\title{
A review on the modeling and simulations of solid-state diffusional phase transformations in metals and alloys
}

\author{
Xueyan Liu, Hongwei $\mathrm{Li}^{*}$, and Mei Zhan \\ State Key Laboratory of Solidification Processing, Department of Materials Science and Engineering, Northwestern Polytechnical \\ University, P. O. Box 542, Xi'an, Shaanxi, 710072, PR China
}

Received: 6 March 2018 / Accepted: 16 April 2018

\begin{abstract}
Solid-state diffusional phase transformations are vital approaches for controlling of the material microstructure and thus tailoring the properties of metals and alloys. To exploit this mean to a full extent, much effort is paid on the reliable and efficient modeling and simulation of the phase transformations. This work gives an overview of the developments in theoretical research of solid-state diffusional phase transformations and the current status of various numerical simulation techniques such as empirical and analytical models, phase field, cellular automaton methods, Monte Carlo models and molecular dynamics methods. In terms of underlying assumptions, physical relevance, implementation and computational efficiency for the simulation of phase transformations, the advantages and disadvantages of each numerical technique are discussed. Finally, trends or future directions of the quantitative simulation of solid-state diffusional phase transformation are provided.
\end{abstract}

Keywords: Solid-state diffusional phase transformation / numerical simulation / empirical and analytical models / phase-field models / cellular automata / Monte Carlo models / molecular dynamics methods

\section{Introduction}

Mechanical and functional properties of materials such as metals and alloys are largely dependent on their microstructure characteristics which include specific volume fraction of spatially distributed phases with different compositions and/or crystal structures, the size and morphology of grains with different orientations and their spatial distribution [1,2]. Due to solid-state diffusional phase transformation, which involves nucleation, growth and impingement, directly results in the arrangements of constituent phases with different grains size and morphology, it plays an important role in the production of materials with various microstructures [3]. For better control over the material microstructures and thus tailor the properties of metals and alloys, comprehensively understanding and manipulating the process of solid-state diffusional phase transformations are vital and necessary. However, during the phase transformations process, the microstructure evolution is extremely complex and nonlinear in nature because it is affected by heterogeneous grains size of parent phase, asynchronous transformation in different grains at different times, crystallographic anisotropy, thermodynamics, kinetics, and other factors [4]. Therefore, the numerical simulation techniques that can

\footnotetext{
* email: lihongwei@nwpu. edu.cn
}

compensate for restrictions of experimental conditions and dynamically reconstruct microstructure evolution are increasingly attractive.

At present, the most advanced numerical simulation methods include empirical and analytical models [5-7], phase field models [8,9], cellular automaton models [10-12], Monte Carlo models [13] and molecular dynamics methods [14]. Empirically-based model, which is developed on the basis of the classical Johnson-Mehl-Avrami-Kolmogorov (JMAK) theory, plays a key role in simulation and prediction of transformations including recrystallization and phase transformation where nucleation, growth and impingement mechanisms operate [15]. Different from empirically-based model, analytical model is developed on the classical physics and phenomenological theory. In general, empirical and analytical models focus more on providing average information such as the average grain size and phase volume fraction of microstructure evolution in macroscale. By contrast, mesoscopic models including phase field models, cellular automaton and Monte Carlo models can reconstruct the evolution of grain morphology and spatial distribution, solute concentration field in addition to giving macroscopic average information [16]. The phase-field method has become an important and extremely versatile technique for simulating solidification, precipitate growth, coarsening, grain growth, martensitic transformations, dislocation dynamics, crack propagation and so on. However, the large 
number of difficult-to-measure phenomenological parameters in phase-field equations and the consumption of huge computing resources greatly decrease the universality of phase-field methods in engineering applications [17]. Compared with phase-field method, cellular automaton technique [18] is more feasible to characterize complex topology changes because of simplicity of formulation, excellent scalability for computer code parallelization and high computational efficiency. Nevertheless, accuracy and reliability of the simulation results for cellular automaton method strongly depend on grid partitioning and switching rules of neighboring cells. The Monte Carlo method [19] is similar to cellular automaton method about computational efficiency, but the lack of physical length and time scales makes the algorithm less attractive. In microscale and nanoscale, the molecular dynamics method is the most representative for characterizing microstructure by tracking the motion of atoms [20].

The present review provides a brief summary of the theoretical research of solid-state diffusional phase transformations and the primary numerical simulation techniques. Subsequently, by comparing these simulation techniques with each other, the advantages and disadvantages of each technique are discussed. Finally, the development directions for future investigations will be proposed.

\section{State of art in solid-state diffusional phase transformations}

Solid-state diffusional phase transformations represent a common phenomenon that when temperature field and other external factors change in many alloys, the diffusion of components in grains and interfaces is activated, and then the migration of interfaces takes place. This process is accompanied by nucleation, growth and impingement of new phase, changes of volume fraction and chemical composition of the individual phases, and microstructure evolution such as lamellar precipitates[21,22]. Since the microstructure determines material properties, it is of great theoretical and realistic importance to realize the accurate description of solid-state diffusional phase transformations and the precise control of the process for tailoring the properties and optimizing the hot working process of metals and alloys.

\subsection{Driving force for phase transformation}

During the phase transformations, there exist two typically basic processes: one is the diffusion of components in grains and interfaces responsible for the change in chemical composition, the other is the interface migration responsible for a gradual rearrangement of the lattice of the parent phase into the lattice of the product phase [21]. In many studies [23-25], the phase transformations were assumed to evolve under local equilibrium conditions, in which case the kinetics was controlled by solute diffusion. In other words, the rate of interface migration was assumed infinitely fast [26]. The classical description of diffusion-controlled kinetics is Zener equation [27]. However, the actual lattice

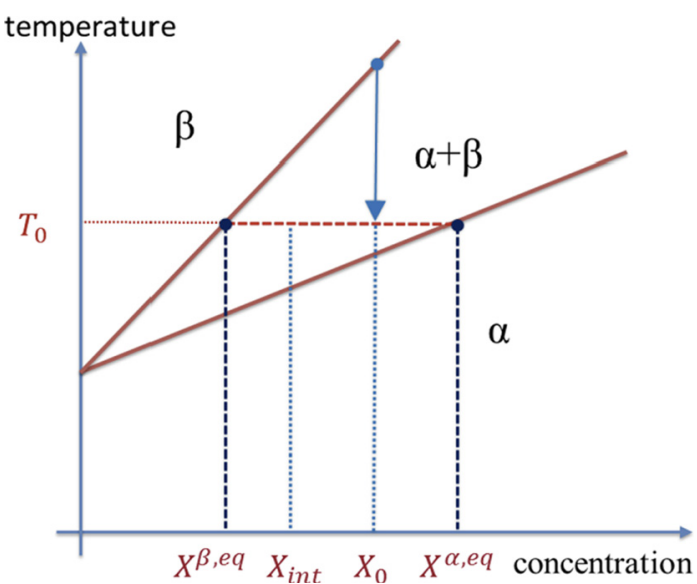

Fig. 1. Schematic representation of the partial phase diagram of a binary A-B alloy.

transformation from the parent phase into the new phase is not an infinitely fast process. Christian [28] assumed that the diffusion velocity was limitless fast and then introduced the concept of interface-controlled kinetics, in that case the interface velocity was proportional to the driving force. At present, the majority of analyses about solid-state diffusional phase transformations are based on one of the diffusion-controlled and the interface-controlled kinetics. However, more and more studies indicate that two control modes are the extreme possibilities for the actual phase transformations [29-31]. Both the diffusivity and interface mobility are not infinite, the phase transformations will be of the mixed-mode character [27]. It means that the driving force on phase interface is partially spent for diffusion and partially for interface migration.

In the following sections, the character of the solid-state diffusional transformation will be demonstrated by examples from a partitioning phase transformation of a binary alloy. Consider a binary solution consisting of two components $\mathrm{A}$ and $\mathrm{B}$. A forms the lattice and $\mathrm{B}$ is the solute. This alloy has two phases that are $\alpha$ (product) phase and $\beta$ (parent) phase. In the two phases, both the crystal structures and mole fractions of $\mathrm{B}$ are different. By taking a $\mathrm{Ti}-\mathrm{Al}$ alloy as example, the $\mathrm{Ti}$ lattice has a bcc structure in $\beta$-phase and a hcp structure in $\alpha$-phase. More $\mathrm{Al}$ atoms can dissolve in $\alpha$-phase than in $\beta$-phase. The phase transformation will occur when the temperature in system decreases and thus a single-phase region $(\beta)$ turns into a two-phase region $(\alpha+\beta)$ (Fig. 1). Initially overall concentration of component $\mathrm{B}$ is given by $X_{0}, X^{\alpha, e q}$ denotes the concentration of component $\mathrm{B}$ in $\alpha$-phase in equilibrium with $\beta$-phase. $X^{\beta, e q}$ denotes the concentration of component $\mathrm{B}$ in $\beta$-phase in equilibrium with $\alpha$-phase. $X_{\beta}$ denotes the concentration of component $B$ in $\beta$-phase far from the interface and $X_{\text {int }}$ denotes the concentration of component $\mathrm{B}$ in $\beta$-phase at the interface. In general, $X_{\beta}$ is assumed equal to $X_{0}$ without taking into account the soft impingement effect. If diffusion of the solute in the growing phase $\alpha$ is ignored, it can be assumed that the concentration in $\alpha$ phase is $X^{\alpha, e q}$. If the concentration of component $\mathrm{B}$ in parent phase and new phase are equal to $X_{0}$, near the interface, the kinetics of the transformation is controlled by 


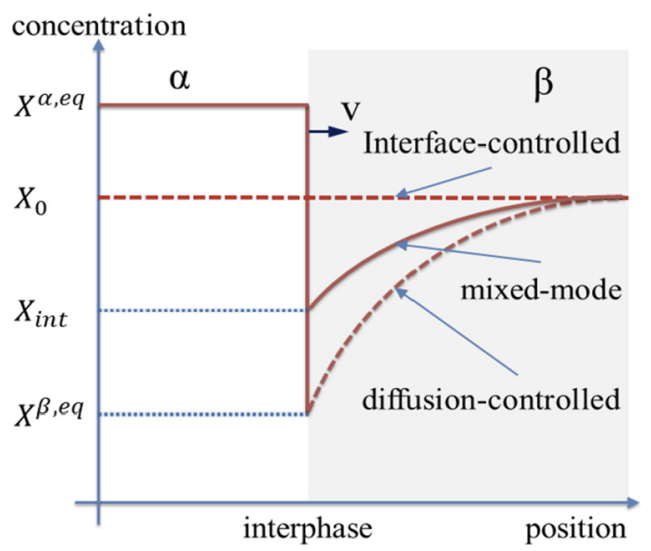

Fig. 2. Planar interface migration and solution partitioning of a binary A-B alloy with initial concentration for component B [26].

interface migration. If the concentration of component B in parent phase and new phase are $X^{\beta, e q}$ and $X^{\alpha, e q}$ near the interface, respectively, the kinetics of the transformation is controlled by diffusion. Actually, the phase transformation is of the mixed-mode character, the concentration of component B in $\beta$-phase at the interface is greater than $X^{\beta \text {, }}$ ${ }^{e q}$ and less than $X_{0}$ (Fig. 2).

Following Hillert [32], the driving force for the phase transformation is equal to the change of Gibbs energy in the system before and after transformation. The change of Gibbs energy for forming per mole of $\alpha$ phase (Fig. 3) is

$$
F_{c h e m}=\left(1-X^{\alpha, e q}\right)+X^{\alpha, e q}\left(\mu_{A}^{\beta}-\mu_{\beta}^{\alpha}\right) .
$$

The Gibbs energy is available for (a) the trans-interface diffusion $\left(F_{\text {Chem }}^{m}\right)$ which means the diffusion of solute in interface, (b) the diffusion of solute in parent phase near the interface and the change in lattice $\left(F_{\text {Chem }}^{t}\right)$. The driving force for trans-interface diffusion is proportional to $\mu_{B}^{\beta}-\mu_{B}^{\alpha}[33]$.

Svoboda [21] et al. assumed that the interface was negligibly thin relative to the grain size, and the diffusion coefficients of solute in interface were slightly larger than those in parent phase. Therefore, the diffusive fluxes in interface were supposed as practically same as in parent phase near the interface. There is no reason to expect any discontinuity of chemical potential of the solute across the interface. Thus, in the approximation of the sharp interface,

$$
\mu_{A}^{\alpha} \neq \mu_{A}^{\beta}, \mu_{B}^{\alpha}=\mu_{B}^{\beta} .
$$

As a consequence,

$$
F_{\text {chem }}^{t}=0, F_{\text {chem }}=F_{c h e m}^{m}=\left(1-X^{\alpha, e q}\right)\left(\mu_{A}^{\beta}-\mu_{A}^{\alpha}\right)
$$

In Figure 3, it can be found that the driving force $F_{\text {chem }}$ $\left(F_{\text {Chem }}^{m}\right)$ reaches the maximum as the concentration $X_{\text {int }}$ of solute B is equal to $X_{0}$ while the driving force decreases to 0 as $X_{\text {int }}$ approaches $X^{\beta, e q}$. Hence, it is reasonable to write $F_{\text {chem }}$ as a function of $\left(X_{\text {int }}-X^{\beta, e q}\right)$. Eriksson [34] evaluated the $f\left(X_{\text {int }}-X^{\beta, e q}\right)$ by thermodynamics software e.g., CHEMSAGE, and the function $f$ is linear for a sufficiently

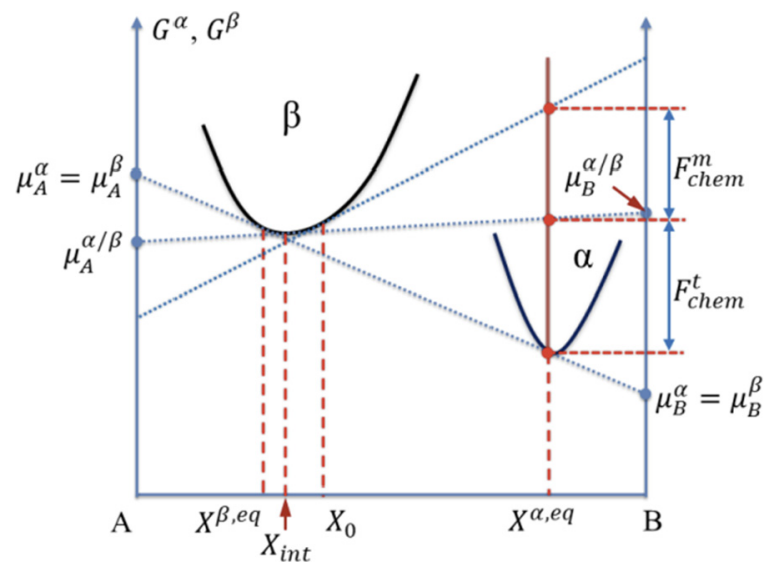

Fig. 3. Schematic representation of molar Gibbs energy for the $\beta \rightarrow \alpha$ transformation in an $\mathrm{A}-\mathrm{B}$ alloy in order to derive the driving force acting on interface $[32,36]$.

large interval of $X_{\text {int }}$ and $X^{\beta, e q}$. Therefore, a factor $\tilde{\beta}$ was introduced in the function,

$$
F_{\text {chem }}=\tilde{\beta}\left(X_{\text {int }}-X^{\beta, e q}\right)
$$

\subsection{Nucleation of new phase}

It is extremely short in time for nucleation process of the new phase, and the nucleus in nanoscale is tiny. Therefore, to directly observe and analyze the nucleation process is particularly difficult by experiments. Aiming at this problem, in the first half of the 20th century, Gibbs [35], Becker [36], Cahn and Hilliard [37,38] conducted systematic theoretical analysis for nucleation and then developed a series of nucleation theoretical models to derive the nucleation rate, activation energy barrier, size and equilibrium shape of the critical nucleus.

\subsubsection{Classical nucleation theory}

The classical nucleation theory is based on the assumption that the thermodynamic properties of a nucleus are uniform and the same as the equilibrium bulk counterparts within the nucleus, and that the interface between the nucleus and the parent phase is sharp [39]. In the classical nucleation theory [22], the nucleation rate is dependent on the number of critical nucleus determined by the activation energy barrier of heterogeneous nucleation and the jump frequency of solute atoms across interface which follows Arrhenius equation. Thus, the nucleation rate is [40]

$$
\dot{N}(T(t))=C \omega \exp \left(-\frac{\Delta G_{h e t}^{*}(T(t))+Q_{N}}{R T(t)}\right),
$$

where $C$ is the density of nucleation sites of new phase, $\omega$ is the frequency factor, $\Delta G_{\text {het }}^{*}(T(t))$ is the activation energy barrier of heterogeneous nucleation and $Q_{N}$ is the activation energy of atomic migration across interface. The classical nucleation models include the continuous nucleation models, pre-existing nuclei models, Avrami nucleation models and mixed nucleation models. 


\subsubsection{Continuous nucleation}

When the undercooling or the overheating is very large, $\Delta G_{\text {het }}^{*}(T(t))$ can be neglected and the nucleation rate per unit volume is only determined by the atomic mobility for transport through the interface. The nucleation rate is [41]

$$
\dot{N}(T(t))=N_{0} \exp \left(-\frac{Q_{N}}{R T(t)}\right),
$$

where $N_{0}$ is the temperature-independent nucleation rate, $Q_{N}$ is the activation energy for the jumping of atoms through the interface between the particle of critical size and the matrix. In this mode of nucleation, it is assumed that the nucleation rate is constant at constant temperature and the number of nuclei is zero at the beginning of transformation. The continuous nucleation often operates in the crystallisation of amorphous alloys.

\subsubsection{Saturation by pre-existing nuclei}

In contrary to the assumptions of continuous nucleation, Cahn supposed that preferential nucleation at grain boundaries, edges or corners caused saturation of the nucleation sites at the beginning of transformation and the further nucleation rate is zero. The saturation of the nucleation sites is depended predominantly on the degree of undercooling or superheating. In this case, the nucleation rate is $[42]$

$$
\dot{N}(T(t))=N^{*} \delta\left(\frac{T(t)-T_{0}}{\varphi}\right),
$$

where $N^{*}$ is the number of (pre-existing) nuclei per unit volume, $\delta\left(\frac{T(t)-T_{0}}{\varphi}\right)$ denotes Dirac functions and $\varphi$ is the constant heating/cooling rate.

\subsubsection{Avrami nucleation}

According to this nucleation mechanism, the particles of supercritical size (nuclei) are formed from the particles of subcritical size, so the total number of particles of subcritical and supercritical size is constant $[43,44]$. In this case, the nucleation rate is

$$
\dot{N}(T(t))=-\dot{N}_{s u b}=\lambda N^{\prime} \exp \left(-\int_{0}^{t} \lambda d \tau\right)
$$

where $\lambda$ is the frequency of becoming the particles of supercritical size (nuclei) from the particles of subcritical size, which follows Arrhenius equation. N' is the number of subcritical particles at $t=0$.

\subsubsection{Mixed nucleation}

In practice, the nucleation process may involve a variety of different nucleation mechanisms, which is so-called mixed nucleation phenomenon. It often represents a combination of the pre-existing nuclei and continuous nucleation modes. The nucleation rate is equal to some weighted sum of the nucleation rates according to continuous nucleation and pre-existing nuclei [41].

\subsubsection{Non-classical nucleation theory of Cahn and Hilliard}

In contrast to the classical theory, the non-classical nucleation theory, which is developed by Cahn and Hilliard [37] firstly, is based on the diffuse-interface description of the interfaces (or gradient thermodynamics). Poduri and Chen [39] extended the non-classical theory of Cahn and Hilliard to describe the phase transformations involving both compositional and structural changes associated with ordering. Heo and Chen [45] gave a briefly review on the recent advances in modeling and predicting nucleation during solid-phase transformations based on the diffuseinterface or non-classical description of critical nucleus profiles.

\subsection{Growth of new phase}

The growth of new phase in solid-state diffusional phase transformations is the migration of the phase interface in essence. According to the description from section 2.1, there exist three control modes including the diffusioncontrolled mode, the interface-controlled mode and the mixed-mode for the migration of interface. Here, the boundary conditions for non-equilibrium interface [46] and the diffusion-controlled and mixed-mode kinetics of the growth for new phase are given as follows.

\subsubsection{Interface boundary conditions}

At the interface, the flux $J_{\alpha / \beta}$ of solute $\mathrm{B}$ atoms into the parent phase by the migration of interface is

$$
J_{\alpha / \beta}=\frac{1}{V_{m}}\left(X^{\alpha, e q}-X_{\text {int }}\right) V(t),
$$

where $V(t)$ is the velocity of the moving interface, $V_{m}$ is the molar volume of element $\mathrm{A}$ in parent phase.

In the parent phase near the interface, the diffusive flux $J_{\text {diff }}$ of solute B atoms along its gradient is

$$
J_{\text {diff }}=-\left.\frac{1}{V_{m}} D \frac{\partial X}{\partial x}\right|_{x=L} .
$$

Here, $D$ is the diffusivity of solute $\mathrm{B}$ atoms in parent phase, $L$ is the position of interface and $\left.\frac{\partial X}{\partial x}\right|_{x=L}$ is the solute concentration gradient in parent phase near the interface.

Since the solute B atoms cannot accumulate at the interface, $J_{\alpha / \beta}=J_{\text {diff. }}$ The velocity of moving interface is acquired by

$$
V(t)=\left.\frac{D}{\left(X_{i n t}-X^{\alpha, e q}\right)} \frac{\partial X}{\partial x}\right|_{x=L} .
$$

\subsubsection{Diffusion-controlled growth kinetics}

For the diffusion-controlled phase transformation, in equation (10), $X_{i n t}$ is equal to $X^{\beta, e q}$. In order to solve the velocity of moving interface, the solute concentration gradient $\left.\frac{\partial X}{\partial x}\right|_{x=L}$ is prerequisite. Since the interface is moving, $\left.\frac{\partial X}{\partial x}\right|_{x=L}$ is extremely difficult to solve. However, the distance $L(t)$ of interface migration is proportional to $\sqrt{D t}$, the so-called "constant-radius" approximate solution $[47,48]$ and the exact solution of Carslaw and Jaeger [49] were proposed, which was subsequently examined by 
Aaron [50] et al. In a strict sense, the so-called "constantradius" approximate solution is valid only for very small supersaturation $\Omega(\Omega<0.1)$. For larger values of $\Omega$, the exact solution of Carslaw and Jaeger is available [51].

In case of the "constant-radius" approximate solution, the distance of interface migration is

$$
L(t)=\sqrt{2 \Omega D t},
$$

and the supersaturation can be write

$$
\Omega=\left(X_{\beta}-X_{i n t}\right) /\left(X^{\alpha, e q}-X_{i n t}\right) .
$$

In general, $X_{\beta}$ is assumed as equal to $X_{0}$ without taking the soft impingement into account.

In a similar way, the exact solution of Carslaw and Jaeger, in which the supersaturation $\Omega$ is replaced by a parameter $\lambda$, is expressed as

$$
L(t)=2 \lambda \sqrt{D t} .(14)
$$

The parameter $\lambda$ has a relation with the supersaturation $\Omega$ as

$$
\left\{\lambda^{2}, \exp \left(\lambda^{2}\right)\right\} \cdot\left[\exp \left(-\lambda^{2}\right)-\lambda \pi^{\frac{1}{2}} \operatorname{erfc}(\lambda)\right]=\frac{\Omega}{2} .
$$

Thus, the velocity of moving interface can be written as

$$
V(t)=d L / d t .
$$

\subsubsection{Mixed-mode growth kinetics}

For the mixed-mode phase transformation, in equation (10), $X^{\beta, e q}<X_{\text {int }}<X_{\beta}$. In general, $X_{\beta}$ is equal to $X_{0}$ without taking into account the soft impingement. In order to solve the velocity of moving interface, not only the solute concentration gradient $\left.\frac{\partial X}{\partial x}\right|_{x=L}$, but also the concentration $X_{\text {int }}$ as a function of time $t$ should be acquired. To tackle the evolution problem of solute concentration field in parent phase near the interface, Sietsma [26] et al. introduced a Zener-like approach and Svoboda [21] proposed an analytical technique by developing a finite difference numerical method.

In the Zener-like approach, Sietsma et al. assumed the concentration gradient $\left.\frac{\partial X}{\partial x}\right|_{x=L}$ was constant and concentration gradient field extended over a distance $l$ into the parent phase. Thus,

$$
\left.\frac{\partial X}{\partial x}\right|_{x=L}=\frac{X_{\text {int }}-X_{\beta}}{l} \text {.(17) }
$$

When the interface migrates to the position $L$, the new phase forms with a volume $V^{\alpha}$ and an interfacial area $A^{\alpha}$. On account of conservation of B-atoms at the interface,

$$
l=\left(\frac{2 V^{\alpha}}{A^{\alpha}}\right)\left(\frac{X^{\alpha, e q}-X_{\beta}}{X_{\beta}-X_{\text {int }}}\right)
$$

According to Christian [52], the interface is proportional to the driving force

$$
V(t)=M F_{\text {chem }} \cdot(19)
$$

Here, $M$ is the effective interface mobility with taking the effects of solute drag or transformation strain into account.

By substituting (4) into (18), plugging (16) and (17) into (10), and then combining (18) with (10), the concentration $X_{\text {int }}$ will be acquired that

with $\Delta X_{\beta}=X^{\alpha, e q}-X_{\beta}$, the parameter $Z$ is given by

$$
Z=\frac{D}{M \tilde{\beta}} \frac{A^{\alpha}}{V^{\alpha}}
$$

According to Svoboda [21], the evolution of the interface is governed by the following system of equations: the bulk diffusion equation

$$
\frac{\partial X}{\partial t}=\frac{\partial}{\partial x}\left(\mathrm{D} \frac{\partial X}{\partial x}\right)
$$

and the initial condition is

$$
X(x, t=0)=X_{0} .
$$

By combining (4),(10) with (18), the boundary conditions at the phase interface are obtained as

$$
\begin{gathered}
\left\{\frac{\partial X}{\partial x}=-\frac{1}{V_{m} D} M X \tilde{\beta}\left(X-X^{\beta, e q}\right),\right. \\
\text { with } X=X_{\text {int }} \quad x=L(t) \quad x \vec{\infty}, X=X_{0} .
\end{gathered}
$$

For the differential equation (21), by considering the boundary initial conditions (Eq. (23)), a nonlinear Volterra integral equation is derived for the mole fraction $X_{\mathrm{int}}$, which is simplified as

$$
\begin{aligned}
X_{\text {int }} & =X_{0} \\
& -\sqrt{\frac{D}{\pi}} \int_{0}^{t} \frac{1}{V_{m} D} M X_{i n t} \tilde{\beta}\left(X_{i n t}-X^{\beta, e q}\right) \frac{d \tau}{\sqrt{t-\tau}} .
\end{aligned}
$$

By plugging (24) and (4) into (8), the velocity of moving interface is

$$
V(t)=\frac{1}{V_{m}} M \tilde{\beta}\left(X_{0}-X^{\beta, e q}\right)\left(\frac{1-2 M \tilde{\beta} X_{0}}{V_{m} \sqrt{D \pi}} \sqrt{t}\right) .
$$

\subsubsection{Impingement of grains in new phase}

For the phase transformations that proceed by the formation of nuclei and the growth of these nuclei, it is impossible that a new phase grows into its parent phase without any limit. In other words, the collision process between two growing neighboring grains is inevitable, which results in obstructing growth of the neighboring grains. It is so-called impingement phenomenon. In

$$
X_{\text {int }}=\frac{Z X_{\beta}+X_{\beta}\left(X^{\alpha, e q}+X^{\beta, e q}\right)+\sqrt{\left(Z X_{\beta}+X_{\beta}\left(X^{\alpha, e q}+X^{\beta, e q}\right)\right)^{2}\left(Z+2 X_{\beta}\right)\left(Z X_{\beta}^{2}+2 X_{\beta} X^{\alpha, e q} X^{\beta, e q}\right)}}{Z+2 X_{\beta}},
$$


general, two impingement modes may occur. One is the hard impingement considering only the geometric factor, and the other is the soft impingement involving a compositional change. In the case of soft impingement, when the diffusion fields start to overlap, the phase transformation will slow down $[53,54]$. However, for the solid-state diffusional phase transformations, soft impingement has been found to prevail, and there usually are wide diffusion layers around the growing nuclei impinging on each other $[55,56]$. Initially a mean field approximation $[27,57]$ was used to take into account the soft impingement effect in the diffusion-controlled growth models. However, it is available for mean field approximation in an assumption that a nucleus grows in isolation, which doesn't consider any effect of the neighboring nuclei on the diffusion field. Subsequently, to deal with the overlap of diffusion fileds for the neighbouring grains in more strict ways, many diffusion-controlled models [58,59] and mixedmodes models $[60,61]$ have been developed to describe the soft impingement effect more accurately.

\section{Numerical simulation techniques for solid- state phase transformation}

\subsection{Empirical and analytical models}

In studies of solid-state diffusional phase transformations involving nucleation, growth and impingement, the classical empirical and semi-empirical models, such as Johnson-Mehl-Avrami- Kolmogorov (JMAK) approach $[52,62]$ or modified JMAK approach (JMAK-like equations) [63,64] and physical analytical models (for example, Zener-Hillert equation, the thermal activation principle of interface of Christian), always play important roles. The years 1937 through 1941 mark an important period in the history of metallurgy. In these years, Johnson and Mehl, Avrami, and Kolmogorov derived expressions for the transformed fraction $f$ as a function of time $t$ in the isothermal transformations [43,65]. The famous JohnsonMehl-Avrami- Kolmogorov (JMAK) equation is

$$
f=1-\exp \left\{-\left[k_{0} \exp \left(-\frac{Q}{R T}\right)\right] t^{n}\right\},
$$

but the JMAK formula can only be validated when the auxiliary conditions imposed in the original equation are not violated, such as: either pure site saturation at time $t=0$ or pure continuous nucleation, large undercooling or overheating for forming high driving force, and randomly dispersed nuclei which grow isotropically $[65,66]$. In other words, the growth exponent $\mathrm{n}$, the effective activation energy $\mathrm{Q}$ and the pre-exponential factor $k_{0}$ are constant relative to time and temperature. By introducing additivity rule into the original JMAK equation, the application the JMAK-like equation was extended to the non-isothermal transformation. However, the kinetic parameters remain constant [67], which follows so called "iso-kinetics". In practice, due to nonrandom distribution of nuclei [68], anisotropic growth [69] and the temperature dependence of the diffusion coefficient and the solute concentration at interface [7], these models which follow so called "isokinetics" are invalid for solid-state diffusional isothermal or non-isothermal phase transformations. Recently, since Cahn [70] first proposed the modified JMAK model with considering nucleation in grain boundary, edge and corner, lots of modified JMAK models by introducing the transient nucleation [71], the spatially correlated nucleation [72], the mixture of nucleation modes, the necessarily associated size-dependent growth [73] or the non-parallel anisotropic growth [74], and the hard or soft impingement, were derived. Shneidman et al. [75] developed a modified JMAK model by considering the effects of transient nucleation, size-dependent growth rate and their correlativity. Tomellini [76] introduced the impingement factor into the JMAK equation to tackle two processes: spatially correlated nucleation with constant nucleation rate and instantaneous cluster growth where cluster overgrowth prevails. By considering variable kinetic parameters (i.e., $n(t), \mathrm{Q}(t)$, $k_{0}(t)$ for isothermal case; $n(T), Q(T), k_{0}(T)$ for nonisothermal case) and the mixture of site saturation, continuous nucleation and Avrami nucleation, Liu et al. [77] proposed a general, flexible and modular analytical model, which expounded the influence of the different nucleation and growth modes on the time and temperature dependencies of the transformation rate and the kinetic parameters. For the treatment of the anisotropic effect, up to now, there exist two approaches : one is the phenomenological extension of JMAK-like formulation by adding (one or more) new variables which provide freedom to improve the agreement where anisotropic growth occurs [15], the other is devoting more attention to the derivation of analytical description with physically realistic variables (e.g. the growth rate anisotropy and the orientation) according to the physical essence of anisotropic effect [78]. In order to describe the mutual blocking of growing particles determined by all relevant orders due to the anisotropic effect, Kooi [74,79] developed an analytical model based on Monte Carlo (MC) simulations by incorporating anisotropic growth into the Johnson-Mehl-Avrami-Kolmogorov models. With respect to the effect of the thermal history, Song [80] proposed a generalized iso-kinetics conditions and additivity rule involving the thermal history-dependent instantaneous reaction rate by introducing a thermal history-related function. So far, a proper analytical approach in the spirit of JMAK-like theory, which can comprehensively consider the different modes of nucleation, growth and impingement, the interactions between nonrandomly dispersed nucleation and anisotropic growth (involving single grain or multiple grains) in solid-state phase transformation, has not yet been developed. In physical analytical models, Zener-Hillert [81] equation established the theoretical foundation for the diffusioncontrolled growth kinetic of lamellar phase. Subsequently, Ivantsov [82], Horvay and Cahn [83] derived a more generally applicable equation with taking into account the elliptic-parabolic or spherical surface for the tip of new phase. Trivedi [84] introduced the capillary phenomenon and interface dynamics into the equation of Ivantsov. Since the anisotropic effect of strain energy, diffusion and crystallography were not considered, these models were still incompetent to predict solid-state diffusional phase 

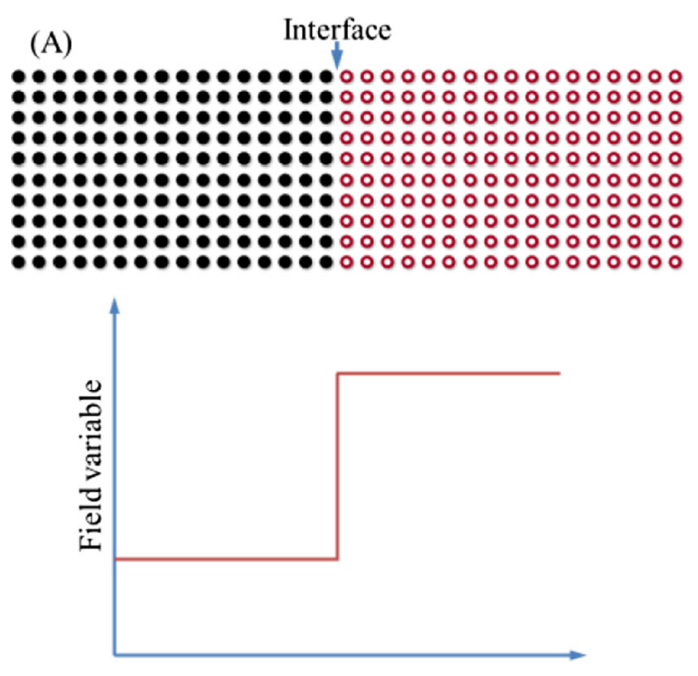

Distance
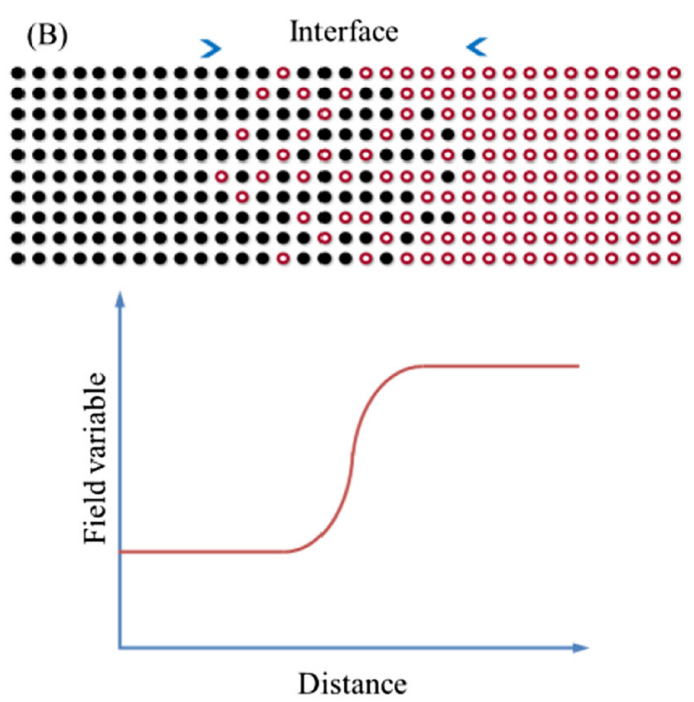

Fig. 4. (A) Sharp interface: properties are discontinuous at the interface; (B) Diffuse interface: properties evolve continuously between their equilibrium values in the neighboring grains[17,36].

transformations accurately. Over all, much effort still should be paid to study the kinetic models of solid-state diffusional phase transformations with integration of different factors and involving structure evolution.

\subsection{Phase-field models}

The phase-field approach has emerged as an important and extremely versatile technique for simulating microstructure evolution at the mesoscale level. Initially, the phasefield models were used to simulate dendrite formation in solidification process. Subsequently, with the increasing computer power, the applicability of phase-field models was extended to solid-state transformations, grain growth, dislocation dynamics, crack propagation, electromigration and solid-state sintering and so forth [17].

In the conventional modeling techniques for phase transformation, the regions separating the compositional or structure domains are treated as mathematically sharp interfaces and the multi-domain structure is described by the position of the interfacial boundaries (Fig.4(A)). These models are also called sharp-interface models. The local interfacial velocity is determined by part of boundary conditions or the driving force on the interface. Due to these approaches involve explicit tracking of the interface position $[17,85]$, it becomes difficult to simulate complex microstructure in twodimension or three-dimension. Therefore, the phase-field modeling, which is able to predict the evolution of arbitrary complex grain morphologies without any presumption on their shape or mutual distribution and explicitly tracking the of the interface position, is very popular. The principal characteristic of phase-field model is the diffuseness of the interface between two phases, so it is sometimes also called diffuse interface models. The view of diffuse interface dates back to van der Waals [86] and Cahn \& Hilliard [37]. Chen, Wang, and Khachaturyan $[87,88]$ is first to introduce the diffuse-interface (Fig.4(B)(B)) into solid-state phase transformation modeling. About the historical evolution of diffuseinterface models and the basic concepts of phase-field models, Moelans [17] gave a comprehensive introduction. In phase-field models, the transformation is described by the temporal and spatial evolution of a set of conserved (for concentration field) and non-conserved (involving information on local structure and orientation) field variables which are continuous across the interfacial regions separating the neighboring phases or structures. The evolution of these field variables are governed by the Cahn-Hilliard nonlinear diffusion equation [37,38] and the time dependent Ginzburg-Landau relaxation equation $[89,90]$.

In solid-state diffusional phase transformation, microstructures have the thermodynamically unstable features. The driving force of the temporal and spatial evolution of the microstructure usually consists of a reduction in the bulk-chemical free energy $\left(F_{\text {bulk }}\right)$, a decrease of the total interfacial energy $\left(F_{\text {int }}\right)$ between different phases or between the neighboring grains with different orientation in the same phase, the relaxation of the elastic-stain energy $\left(F_{\text {elast }}\right)$ generated by the lattice mismatch and external energies $\left(F_{\text {appl }}\right)$ due to applied stress, temperature, electrical, and magnetic fields and so on [91]. Thus, the total free energy of a system is

$$
F_{\text {tot }}=F_{\text {bulk }}+F_{\text {int }}+F_{\text {elast }}+F_{\text {appl }} .
$$

In phase-field models, the total free energy [92] described by a set of conserved $\left(c_{1}, c_{2}, \ldots\right)$ and nonconserved $\left(\eta_{1}, \eta_{2}, \ldots\right)$ field variables can be written as

$$
\begin{aligned}
F_{\text {tot }} & =\int\left[f \left(c_{1}, c_{2}, \ldots, c_{n}, \eta_{1}, \eta_{2} \ldots \eta_{\mathrm{p}},+\sum_{i=1}^{n} \alpha_{i}\left(\nabla_{c i}\right)^{2}\right.\right. \\
& \left.+\sum_{i=1}^{3} \sum_{j=1}^{3} \sum_{k=1}^{p} \beta_{i j} \nabla_{i \eta k} \nabla_{j \eta k}\right] d^{3} r+\iint G\left(r-r^{\prime}\right) d^{3} r d^{3} r^{\prime},
\end{aligned}
$$


where $f$ is the local free-energy density, $\alpha_{i}$ and $\beta_{i j}$ are the gradient energy coefficients. The evolution of conserved and non-conserved field variables can be obtained by solving the following the Cahn-Hilliard nonlinear diffusion equation [37] and the Ginzburg-Landau relaxation equation [90],

$$
\begin{aligned}
& \frac{\partial c_{i}(r, t)}{\partial t}=\nabla M_{i j} \nabla \frac{\delta F_{t o t}}{\delta c_{j}(r, t)} . \\
& \frac{\partial \eta_{p}(r, t)}{\partial t}=-L_{p q} \nabla \frac{\delta F_{t o t}}{\delta \eta_{q}(r, t)},
\end{aligned}
$$

here, $M_{i j}$ is related to the mobility of atom or interface and $L_{p q}$ is the symmetrical matrix of dynamic structural operator related to the non-conserved field variables. Therefore, in phase-field models, to accurately describe the microstructure evolution in solid-state diffusional phase transformation including nucleation, anisotropy growth, hard or soft impingement needs to establish the appropriate correlation functions of the field variables and the phenomenological parameters for reflecting aforementioned effects.

In recently, many phase-field models, incorporated with nucleation phenomena [93-95], anisotropy effects [96-99] and elasticity/plasticity [100-104] and macroscopic behaviours [105], have been proposed for better quantitatively predicting the microstructure evolution in solid-state diffusional phase transformations. Zhang, Chen and Heo [96] developed a phase-field model combined with the minimax technique to predict the morphology of critical nuclei during solid-state diffusional phase transformation in both two and three dimensions, which took into account the anisotropic interfacial energy as well as the anisotropic long-range elastic interactions. Then, by incorporating with diffuse-interface critical nuclei in phase-field simulations through the explicit nucleation algorithm, the temporal growth kinetics of the introduced nuclei were obtained [95]. Mecozzi et al. [106] have successfully performed two- and three-dimensional simulations of a grain growth during austenite-to-ferrite transformation by the multi-phase-field (MPF) method, where the interfacial energy and mobility of all interfaces were assumed to be constant. Because the nucleation typically takes place on a much smaller length scale than the further growth of new phase, as above, lots of phasefield models can only be used to study the one of nucleation or growth. To sample frequently enough to observe nucleation would require an impractically large number of integration cycles to be performed in order to observe growth kinetics in real alloys, Simmons et al. [107] introduced a Poisson seeding algorithm into the Phase Field equations instead of the Gaussian-distributed Langevin noise terms for simulating nucleation. Wang [108] used the quantitative phase field method to study the concurrent nucleation and growth in a diffusioncontrolled phase transformation. However, in their works the growth behavior and morphology of simulated grains are isotropic, which is unrealistic in actual phase transformation. Therefore, a series of phase-field models, integrated with the anisotropy growth, have been developed. Yamanaka [109] used a multi-phase-field model with considering the dependence of the interfacial energy and mobility on phase and crystal orientation to simulate growth of the ferrite. Wei [97] used the Loginova phase-field model with introducing a highly anisotropic function related to the anisotropy strength of the interfacial energy and interface thickness, and the KimKim-Suzuki (KKS) phase-field models [S.G. Kim, W.T. Kim, T. Suzuki Phys. Rev. E, 60 (1999), pp.7186-7197] with a thin interface thickness which could enlarge the simulation domain to a large dimension, to simulate the growth of widmanstatten ferrite from grain boundary allotriomorphic ferrite with perturbed interface during isothermal austenite-ferrite transformation. In addition, the effect of elasticity/plasticity resulted from elastic or even plastic deformations should not be neglected. Recently the phase field models, containing the description of elastoviscoplastic material behaviour of the phases combined with diffusion and phase transformation, can be classified into two main groups of theories [110-112]. One is the interpolation models relying on a single set of constitutive equations and interpolation of material parameters, the other is the homogenization models based on the mixture of free energy and dissipation potentials associated with distinct behaviors of the phases. These models have been applied to study the mechanical effect on the diffusion-controlled growth of the precipitates.

Furthermore, for complex multicomponent systems, the phase-field models coupled with thermodynamic and kinetic databases were developed to put more physics into the expansion of the free energy function in the GinzburgLandau equation. Steinbach [113] discussed details of a direct coupling between the Multiphase-Field method and the CALPHAD method and used the CALPHAD - coupled Phase-Field model to simulate solidification microstructure formation in Ni-base superalloys. Wang et al. [114] presented a Multiphase-Field model with inputs from CALPHAD thermodynamic and DICTRA kinetic databases and simulated the growth and dissolution of a precipitates in $\mathrm{Ti}-\mathrm{Al}-\mathrm{V}$ on experimentally relevant length and time scales.

In addition, a number of the phase-field simulations for solid-state phase transformations have been conducted in three-dimensions (3D). In the complex phase transformations, due to that the two-dimensional (2D) models cannot simulate the basic microstructural features, the three-dimensional (3D) simulations which show a realistic representation of the actual microstructure become more atrractive. M.Militzer [115] used the phase field method to simulate the austenite-to-ferrite phase transformation in steel in three-dimensional (3D) space. It was found that the effect of the exact morphology of the initial 3D austenitic microstructure on the kinetics was shown to be small and three-dimensional (3D) simulations predict slower transformation rates compared with two-dimensional (2D) simulations. Cui [116] developed a three-dimensional, non-isothermal phase-field model to systematically investigate the interactions between the temperature, stress, and microstructure fields in shape memory alloys. 
As previously mentioned, the phase-field model exhibits the tremendous capability of quantitatively predicting the complex microstructure evolution in solid-state phase transformation. However, for phase-field models [17], there are two major difficulties in achieving more precise quantitative description of the real phase transformation with the coexistence of different nucleation modes, mixed growth mode, anisotropy growth, impingement effect and elasticity/plasticity effect. First, phase-field models contain a great many of phenomenological parameters related to the thermodynamic properties, phase composition, the interfacial structure, the diffusion of solute elements, and the elastic/plastic properties of the coexisting phases. Most of these parameters are difficult to measure. Second, in actual, the width of the interface is several orders smaller than the microstructure features such as grains and precipitates, so massive computer resources will be consumed by resolving the evolution of phase-field variables at the interface and also covering the system with realistic dimensions. Aiming at the problem of excessive computation times and insufficient computer memory, using interface field [117] especially adaptive grids providing a local remeshing at the interface have turned out to be beneficial [118]. For solid-state phase transformation, the Fourier-spectral method with semi-implicit time stepping is usually more appropriate than the technique based on adaptive meshing on account of a large number of grains or precipitates involved [119]. However, these computing techniques are either limited by the number of CPU cores or are too intractable to implement in three-dimensional simulations. Thus, the GPU-based parallel computing technology is extremely attractive in scientific computation owing to the GPU's massive computation capacity and high memory bandwidth. In the past few years, many researchers have successfully applied the GPU parallel computing algorithm into two-dimensional or three-dimensional phase-field simulations of dendrite growth $[120,121]$. To increase the system size towards relevant dimensions, the width of the diffuse interface must be taken artificially large. Much effort has been made in developing phase-field models that allow changing the interface width without affecting interfacial properties or introducing spurious effects [17]. However, a general solution has not yet been found. Therefore, there will still be increasing efforts to develop the more powerful and feasible large-scale three-dimensional (3D) phase-field model.

\subsection{Cellular automaton models}

Cellular automata are synchronous algorithms, first introduced by von Neumann in 1963, and later on in 1986 by Wolfram to a broader scientific public, which describes the discrete spatial and temporal evolution of complex system by applying local (or sometimes midrange) deterministic or probabilistic transformation rules to the cells of a regular (or non-regular, random) grid with local connectivity $[122,123]$. Since Hesselbarth [123] et al. used the cellular automaton approach for the simulation of recrystallization, in the past several decades, it gradually becomes very promising and popular for
(A)

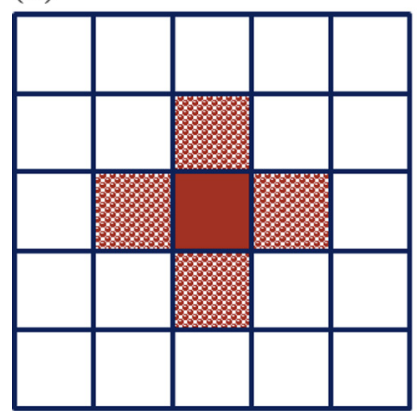

(B)

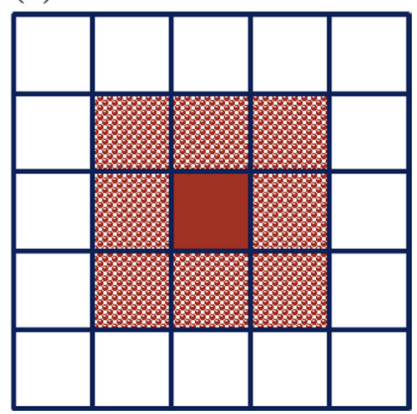

Fig. 5. Neighboring type in cellular automata: (A) von Neumann configuration; (B) Moor configuration.

simulating the microstructure evolution due to its computation efficiency, flexibility in multi-scale bridging, and physical consistency. Cellular automaton method has been successfully performed in two- or three-dimensional modeling and simulations of dynamic and static recrystallization [124,125], grain coarsening [126], solidification [126], solid-state phase transformation [127], micro-shear band and shear band propagation [128], cracks propagation [129], etc. It provides a deeper insight into the mechanism and morphological complexity of them without timeconsuming solution of complicated partial differential equations. So far, all these models can be classified into several main groups that referred as the classical cellular automaton, frontal cellular automaton [130], irregular and shapeless cellular automaton [131], and virtual front tracking model based on cellular automaton [132].

In the classical cellular automata, take the twodimensional model as an example, the entities of the automaton are the types of the neighboring cells (Fig. 5), the state variables of cells in space evolving with time and cell status update rules without any restrictions [133]. A cellular automata simulation for complex microstructure evolution proceeds by discrete simulation steps (namely discrete time steps) consisting of the computation of the new states $\left(t_{0}+\Delta t\right)$ of the all cells based on their current states $\left(t_{0}\right)$, which can be presented as the following function with Moor configuration in two-dimension,

$$
\begin{aligned}
\xi_{i, j}^{t_{0}+\Delta t} & =f\left(\xi_{i-1, j-1}^{t_{0}}, \xi_{i-1, j}^{t_{0}}, \xi_{i-1, j+1}^{t_{0}}, \xi_{i, j-1}^{t_{0}}, \xi_{i, j+1}^{t_{0}}, \xi_{i}\right. \\
& \left.+1, j-1^{t_{0}}, \xi_{i+1, j}^{t_{0}}, \xi_{i+1, j+1}^{t_{0}}\right) .
\end{aligned}
$$

where $\xi_{i, j}^{t_{0}}$ is the value of the state variable of the cell ( $i$, $j$ ) at the time $t_{0}$, and the function $f$ specifies the set of transformation rules which determine the updated state of a cell based on the cell's previous state and the states of all cells in the local neighborhood of that cell. Besides, some conditions at the outer boundaries of the grid such as periodic, reflexive or fixed grid are applied in order to study the infinite target domain by simulating a finite representative region.

The most of cellular automata models, which can be found in literature, are two-dimensional(2D). 2D cellular automata models are simpler and faster in calculation in account of having less elements, connections and complicated algorithms. However, microstructure evolution is 
pointedly three-dimensional phenomena, the results obtained by $2 \mathrm{D}$ cellular automata models cannot always be directly transferred to a real 3D process [130]. Therefore, $3 \mathrm{D}$ cellular automata models attracted a growing number of researches[134,135]. Due to more cells in simulated domain and more neighbours for each cell relative to $2 \mathrm{D}$ models, the 3D models require significantly more memory and time for the calculation, which increases the difficulty in accurately predicting real microstructure evolution.

During the implementation of the classical automaton models, some problems and aspects needing to be improved have been encountered. One of the most significant is the inherent and artificial anisotropy introduced in the computations by the mesh of squared cells, which causes that the preferential growth directions of all the simulated grains are aligned with the mesh axis or at 45 degree and thus leads to the growth kinetics without physical significance. For the problem, Stefanescu et al. [127] developed a cellular automaton model coupled with virtual interface tracking scheme (Fig. 6). In this model, the procedure of constructing virtual sharp interface for capturing new interface cells in a time step is as follows. First, for all interface cells, the length $L_{\phi}$ dependent on the local growth angle $\varphi$ is assumed equal to $f_{s}=1$ (new phase fraction $f_{s}$ ) and measured from the interface cell center along the normal direction of the local interface, which can be written as (Fig. 6(A))

$$
L_{\phi}=\frac{\Delta x}{\max (|\cos (\phi)|,|\sin (\phi)|)},
$$

here cells size is $\Delta x$. The position of the new interface at time $\mathrm{t}+\Delta \mathrm{t}$ can be gauged from the interface cell center along the normal direction of the local interface at a distance proportional to the $f_{s}$ in one cell. And the entire virtual sharp interface at time $\mathrm{t}+\Delta \mathrm{t}$ will be described by connecting the position of new interfaces in all interface cells. Second, the parent phase cells, found inside the area surrounded by the entire virtual sharp interface, are considered captured and will become new interface cells (Fig. 6(B)). Subsequently, after capturing the new interface cells and then updating the state variables, the procedure is repeated again in the next time step.

Another problem is that interface energy and mobility relies on its curvature which is dependent on the orientation of the interface surface, meanwhile, the method of using the symmetrical grid to discretize the surface in such a way that only a very limited number of orientations are represented cannot resolve curvature [131]. For this problem, Janssens et al. [136] first presented the random grid or irregular cellular automaton based on Voronoi cells to solve this problem by local variation of the cell lattice geometry (Fig.7 (A)). Afterwards the shapeless or point cellular automata were developed from the random grid or irregular cellular automaton by Janssens. In this model, shapeless cell shapes are not computed explicitly and only a point position is used, which makes much more flexible in definition of neighborhood (Fig.7(B)).

In addition, no cell state is actually changed until the end of the solution step when the states of all cells are updated simultaneously in the classical cellular automata,
(A)

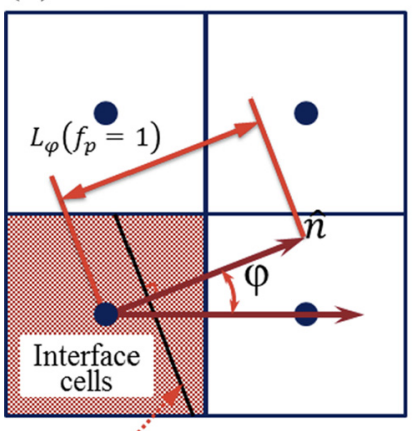

Virtual interface
(B)

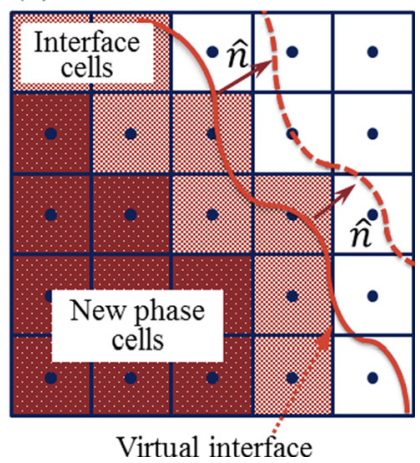

Fig. 6. Illustration of (A) determining the position of sharp virtual interface, and (B) capturing rules for new interface cells $[127,134]$.

which directly influences the calculation time. Much effort has been focused on developing modified cellular automaton models allowing for significant acceleration of the calculation especially in the three-dimensional tasks. In order to only consider the small area near the front of moving interface for growing grains from the calculations in the current step, Svyetlichnyy $[137,138]$ presented the FCA (frontal cellular automata) algorithm. FCA consists of a complex of the millions of cells with the same multi-states automaton (Fig. 8). The series of automaton states includes the initial matrix state $q_{0}$, the frontal cell $q_{1}$, the boundary cell $q_{2}$, the cell inside the grain $q_{3}$, the transient state $q_{4}$ accompanied with calculation of the time delay, nucleation state $q_{5}$, the cell in the edge of the grain $q_{6}$, and the cell in the corner of the grain $q_{7}$. Besides, some conditions used by transition rules respectively are the nucleation condition $I_{0}$, the time delay condition $I_{1}$ which controls grain growth or mobility of interface, the conditions $I_{2}$ and $I_{3}$ which determine nucleus location (on grain boundary, inside the grain, in the edge or corner of the grain), and state change condition $I_{4}$ which refers as that neighboring cells transits from the states $q_{2}, q_{3}, q_{6}, q_{7}$ into the transient state $q_{4}$ for all neighbors of all frontal cells. For the phase transformation, in FCA, the initial states of the cell before transformation can be arbitrary $\left(q_{1}-q_{7}\right)$, and then the conditions $\left(I_{1}-I_{7}\right)$ are responsible for the transformation. The boundary conditions such as periodic displacement conditions and full open conditions are usually used. Relative to the classical cellular automata, the most advantage of FCA is found out allowing for recognition of the changes in cells state without studying their neighborhood.

In the formulation of cellular automaton models for solid-state diffusional phase transformation, each cell represents a volume of real material characterized by certain attributes with a distinct phase or grain, solute concentration and an orientation indictor. There are several state variables with describing certain attributes used on each cell in phase transformation such as the phase state variables, the solute atom concentration variables, the orientation variables, the velocity and displacement variables of the moving interface, and volume fraction variables. The cellular automaton will be in progress by 
(A)

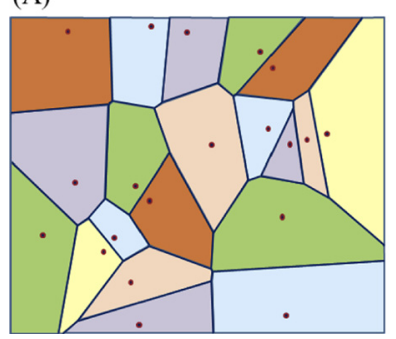

(B)

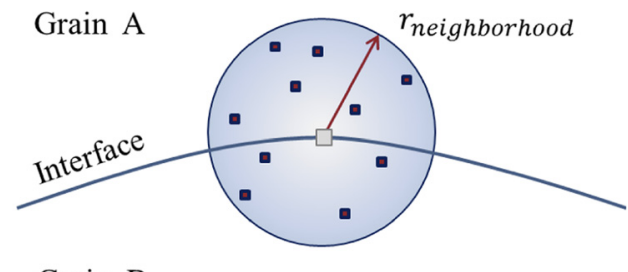

Grain B

Fig. 7. Illustration of (A) cells distribution in a two-dimensional irregular cellular automata based on the Voronoi cells, and (B) a shapeless cell on a curved interface and its spherical neighborhood [121].

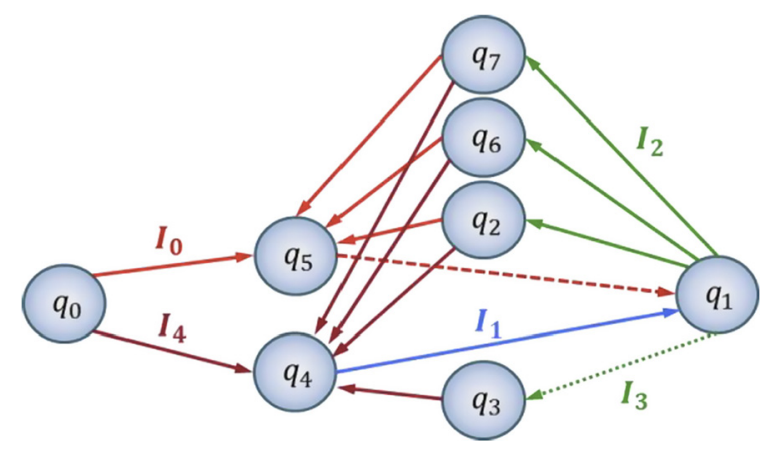

Fig. 8. Illustration of FCA (frontal cellular automata).

synchronously updating these state variables for all lattice cells in each time step, and thus spatial and temporal microstructure evolution is described. In recently, many cellular automaton models for solid-state diffusional phase transformation have been developed to focus on simulating the austenite-ferrite phase transformations in $\mathrm{Fe}-\mathrm{C}$ alloy in which interstitial solute carbon atoms have high diffusion coefficient[139-141]. However, as regard to substitution solid solution like $\mathrm{Fe}-\mathrm{Mn}$ or $\mathrm{Ti}-\mathrm{Al}-\mathrm{Mo}$ alloy, the attractive force occurs from the solute atoms on the moving interface, which is so-called solute drag without existing in interstitial solid solution. According to Cahn [138], the solute atoms have different drag effects in three regimes of the interface velocity. Therefore, the effects of solute drag for solid-state phase transformation in substitution solid solution cannot be neglected. Furthermore, in most of these models, the growth behavior and morphology of simulated grains were assumed to be isotropic [141,142], which was not correspondent with practice. Besides, because the nucleation typically takes place on a much smaller length scale than the further growth of new phase, as with the phase-field models, the cellular automata is not able to simulate the process of different nucleation modes and mixed growth mode occurring in sequence. And last but definitely not least, above mentioned models, the phase transformation is supposed to happen with only the diffusion-controlled or the interface-controlled kinetics in the isothermal condition without considering the effect of the thermal history. A cellular automaton model [143] coupled with Svoboda's analytic solution of diffusional phase transformation was established to divide the diffusion-, mixed- or interface-controlled transformation in isothermal and non-isothermal processes. However, due to the critical conditions could not be obtained as a result of the difficult-to-measure constant factor in the driving force model, and due to only taking into account the cooling ratedependent transformation temperature, the cellular automaton model was not enough to simulate the actual nonisothermal transformation. All in all, much effort should be focused on improving the cellular automata models for better modeling and simulation of the solid-state phase transformation in the future.

\subsection{Monte Carlo models}

MC models were developed from Ising and Potts models of ferromagnetic systems used in statistical physics [144]. Initially they were used to simulate grain growth by Anderson et al. [145], after that they have been widely used to model the microstructure evolution in materials such as the recrystallization and the phase transformation. In Monte Carlo models [146], space is discreted into a set of lattice points onto which a continuum microstructure is mapped at a length scale above that of atoms and below that of grains, similarly to the probabilistic cellular automaton models. The grain boundaries are defined as existing between lattice sites of neighbouring grains. The energy of the system is represented by a Hamiltonian which sums the interfacial energy of the system. The Monte Carlo models simulate curvature-driven grain boundary motion by the random movement of kinks (2D) or ledges (3D) along the grain boundaries controlled by the transition rules. Due to the calculations of grain boundary curvature and energy are ambiguous and the transition rules governing the change of voxel state are often not physically justified, the physical meaning of units of length, time and energy in Monte Carlo models is often unspecified [147]. Nonetheless, the numerical implementation is straightforward and decent computational efficiency can be achieved in the Monte Carlo models, especially since the algorithm is very suitable for parallelization, the Monte Carlo models are still frequently used to model the complex microstructure evolution in materials.

\subsection{Molecular dynamics methods}

Molecular dynamics simulation serves as an essential technique for the investigation of fundamental atomic behaviors in metals and alloys, which was developed by Alder and Wainwright in the late 1950s [148]. It has 
facilitated scientific discovery of interactions among particles or atoms since molecular dynamics method allows us to examine the varying atomic configurations at finite temperatures by incorporating statistical mechanics [149]. The molecular dynamics methods simulate microstructure evolutions from the atomistic point of view by solving the particle trajectories derived from the interatomic forces. Numerical integration of atomic motion is carried out on the interatomic forces, which results in the particle velocity. The velocity of the particles follows the Maxwell-Boltzmann distribution, which is temperature dependent. Then, the particle position is obtained by further integrating the velocity. Molecular dynamics methods have evolved as the powerful tool to investigate the mechanisms of physical phenomena such as nucleation, and nucleus growth which are difficult to observe experimentally [14], [150].

\section{The comparison of different numerical simulation techniques}

As above, the theoretical researches of solid-state diffusional phase transformations and the primary numerical modeling techniques are discussed. All these phase transformation models characterized by complex mathematical formulations present various predictive capabilities. In the following, the advantages and disadvantages of each modeling technique will be discussed in terms of the underlying assumptions, physical relevance, implementation and computational efficiency for the simulation of the solid-state diffusional phase transformations.

Historically, the classical empirical and semi-empirical models represented by Johnson-Mehl-Avrami-Kolmogorov (JMAK) approach were commonly used for simulations of phase transformations. The JMAK formula are valid only in conditions of either pure site saturation at initial moment or pure continuous nucleation, large undercooling or overheating for forming high driving force, and randomly dispersed nuclei which grow isotropically. In these models, all attention is focused on the kinetics while the microstructural aspects are essentially ignored, and thus its only provide average information such as the average grain size and phase volume fraction of microstructure evolution on macroscale. Afterwards, several upgrades of the model with integration of the mixed nucleation modes, anisotropic growth, and the hard or soft impingement effects were proposed. Much effort has been paid on developing the models involving structure evolution, and the approach is still commonly used. Compared with the JMAK approach, the phase field models and the cellular automaton models can reconstruct the evolution of grain morphology and spatial distribution, solute concentration field in addition to giving macroscopic average information.

The phase-field modeling is a mechanistic-based microstructure modeling method, in which phenomenological character and the equations for the evolution of the phase field variables are derived based on general thermodynamics and kinetics principles. It can predict the evolution of arbitrary complex microstructure without any presumption on their shape or mutual distribution and explicitly tracking the of the interface position, meanwhile, provide an insight into controlling mechanisms in solidstate diffusional phase transformations [151,152]. Because a large number of grains or precipitates are involved in solid-state diffusional phase transformation, the Fourierspectral method with semi-implicit time stepping and uniform mesh is usually more appropriate than the technique based on adaptive meshing in phase-field models [17]. However, the phase-field models contain a large number of phenomenological parameters related to the thermodynamic properties, the phase composition, the interfacial structure, the diffusion of solute elements, and the elastic/plastic properties of the coexisting phases, meanwhile, most of these parameters are difficult to measure. Besides, the phase field simulations are more computationally intensive than the JMAK approach and the cellular automata.

Relative to the classical empirical models and the phase-field models, the cellular automata models are more easily implemented without solving complex partial differential equations and its can be used to capture many aspects of the microstructure physics in solid-state diffusional phase transformation. Since the discrete nature of the algorithms is well suited for parallelization, the cellular automaton methods possess the high computational efficiency. However, a precise calculation of the mean curvature which is necessary for grain boundary migration by capillarity remains difficult [98]. Moreover, due to the sharp interface in the cellular automaton models, compared with the phase-field models with the diffuse-interface, the cellular automaton approach is less than accurate to predict solid-state diffusional phase transformation. Besides, limitations of the cellular automata also lie in the dependence on the underlying solution grid and the types of the neighboring cells. In addition, for solid-state diffusional phase transformations, the time step in the cellular automata simulation is virtual or defined as e.g., the ratio between the cell size and maximum interface velocity, or a scaled ratio between the average cell size and the average interface velocity, which is deficient in physical meanings [153].

The Monte Carlo model as usually is very similar to the probabilistic cellular automata model in terms of implementing. Both models describe the discrete spatial and temporal evolution of complex system by applying local transformation rules to the grids with local connectivity. The differences between them lie in the transition rule and the method of updating the state of each cell [154]. In the Monte Carlo models the transition rule is determined by the reduction in local free energy, whereas the cellular automata model is more flexibility to adjust the transition rule for matching kinetics of a particular experimental system. Therefore, the Monte Carlo simulations lack physical length and time scales, and the results are difficult to match to experiment. In the Monte Carlo models sites are chosen at random for updating, whereas in the cellular automata models all sites update simultaneously. Thus, the cellular automata models are generally more efficient than the Monte Carlo models. 
The phase-field models, the cellular automata models and the Monte Carlo models in mesoscale allow the simulation of a relatively large representative volume element that can be composed of thousand to million grains. However, the effects and physical mechanisms are predefined and pre-implemented in these models, it is impossible for them to resolve the mechanisms of phenomena acting at the atomic scale. In this case, the molecular dynamics simulations are utilized to describe atomic interactions to make up for the disadvantage of mesoscopic models. Compared to these mesoscopic models, the representative volume element that can be simulated by the molecular dynamics models in a reasonable time is very limited, which occasionally results in insufficient statistical representativeness and undesired effects of the size of the simulated volume [155]. With increasing computational power, large scale atomistic simulations can be conducted with several million or even billion atom systems, e.g. into studying phase transformation kinetics [156], deformation and fracture in metallic systems [157].

\section{The future prospects}

Although various numerical simulation techniques as above have been successfully used to describe the solidstate diffusional phase transformations, there will also be increasing efforts in the accuracy and efficiency of models. The new trends or future directions for the simulation of solid-state diffusional phase transformation will be expected.

\subsection{Integrating the nucleation phenomena (mixed- modes, nuclear morphology)}

Nucleation of the new phase typically takes place on the much smaller length scale than the subsequent growth of nucleus and it is generally extremely short in time for nucleation process. Moreover, in practice, the distribution of nucleus is not random and there are various nucleation phenomena. It have been found that the nucleation was either related to time like transient nucleation and continuous nucleation, or correlated with space like nucleation in grain boundary, edge and corner at parent phase. In addition, the morphology of the critical nucleus is very complicated by additional contact angle conditions for nucleation occurring at the planar defects. The existing models on mesoscopic scale can only describe a single nucleation mode with ignoring the morphology of nucleus, and thus it is not feasible to consider both the nucleation and the growth at the same time for these models. However, many models represented by the molecular dynamics on microscopic scale and nanoscopic scale are benefited to accurately capture the grain boundary energy and interfacial energy and predict the geometry of the nucleus by tracking motion of atoms. Therefore, future efforts are expected to be focused on coupling between mesoscopic simulation and microscopic or nanoscopic calculations for complex nucleation phenomena in solid-state diffusional phase transformation.

\subsection{Incorporating with the anisotropic effect}

In general, the fundamental process underlying the microstructure evolution in solid-state diffusional phase transformations is the migration of interface or growth of new phase in a broader sense. The kinetics and morphology of grains growth in new phase are determined by the properties of interfaces such as the anisotropic interfacial energy and mobility. Anisotropy of the interfacial energy and mobility reflects the atomistic crystallographic structure of interfaces, meanwhile, the low-energy interfaces structures leads to the faceted interface as well. At present, many findings allow to make a rough estimate successfully for the anisotropic grain boundary energy in case of the same lattice structure and composition. However, with respect to the interfacial energy, the effects of lattices structure and chemical composition also remain largely unexplored and the anisotropy of interfacial mobility is difficult to measure, which make it impossible to accurately predict anisotropic growth of grains in new phase.

\subsection{Introducing the hard or soft impingement effect}

In general, for the phase transformation consisting of nucleation and growth, the collision process between neighboring grains is inevitable. When the impingement occurs, with decreasing of the distance between the edges of neighboring grains, the diffusion fields start to overlap, at the same time, the concentration gradient becomes more flat, the growth rate is predicted to decrease locally and thus the phase transformation slows down. In that case, the growth law cannot be considered as given a priori before collision process since it is a function of the local environment of each grain. However, at present, due to the lack of knowledge on the exact nature of diffusion layers for the hard and soft impingement, the collision process between grains described in the existing models is now less effective than the genuine impingement process.

\subsection{Considering the thermal history}

In the past few decades, various analytical models have been successfully developed to predict the non-isothermal transformations process with the use of the additivity concept in the isokinetic condition. The conventional additivity rule is only valid in the assumption that nonisothermal transformations process is described as the summation of a series of short period isothermal transformation process, which implies that the instantaneous reaction rate only depends on the current state of every short period isothermal transformation process. However, in practice, the diffusion field around new phase during non-isothermal transformation has an intrinsic memory of the thermal history as a result of the temperature dependence of the diffusion coefficient and the local equalized solute concentration at the interphase boundary, which leads to a temperature path-dependent instantaneous growth rate. Therefore, most of analytical models introduced the conventional additivity rule usually overestimate or underestimate the measured extent of 
precipitation reactions. At present, for more accurately predicting solid-state diffusional phase transformations, there has been increasing efforts to be spent on creating the thermal history-related function, and developing a generalized additivity rule which involves the thermal historydetermined instantaneous reaction rate.

\subsection{Coupling with macroscopic deformation}

Solid-state diffusional phase transformations are always not immune to the effect of the elastic deformation and plastic deformation such as the stored energy, the dynamic and static recrystallization, the globularization and the deformation heating and so on during the metal forming process. When the solid-state phase transformation takes place after the elastic-plastic deformation, in other words, the deformation is applied in single-phase region at high temperature or in two-phase region with isothermal conditions and then the cooling treatment is performed at given rate. The stored energy is the main factor for contributing to the driving force of the phase transformation. However, if the solid-state phase transformation process operates simultaneously with deformation, not only the stored energy, the dynamic and static recrystallization, the globularization and the deformation heating have significant influences on the kinetics and crystallography (orientation relationship, morphology) of phase transformation, but also in turn the changes of the volume fractions of constituent phases and grains morphology on the mesoscale affect the deformation resistance on the macroscale. Up till now, due to lack of knowledge on the exact mechanism of the interaction of phase transformation and deformation, few investigations are performed on modeling the phase transformation coupled with macroscopic deformation.

\subsection{Improving computational efficiency and accuracy for quantitative simulation}

Numerical simulation can provide the important insights into the role of special material or process parameters on the volume, shape and spatial distribution of new phase, orientation relationships between new phase and parent phase, or kinetics in solid-state diffusional phase transformation. However, the more versatile and feasible technique for quantitative predicting the microstructure evolution is required both the high computational efficiency and the accuracy for the numerical solution. As is known to all, the more accurate solution of details of the microstructure evolution requires the finer mesh. In contrast, the coarser mesh is more benefit to the high efficient calculation. For the phase-field models, a finite volume or finite element discretization based on adaptive meshing which provides a local re-meshing at the interfaces, or artificially enlarging the width of the diffuse interface is often applied to improving the accuracy of simulation quantitatively and the computational efficiency. However, the problem of excessive computation times and insufficient computer memory is still not solved with the use of these methods. For the cellular automaton models, it is more easily implemented without the excessive computation times and solving complex partial differential equations. However, once coupled with the finite element simulation on macroscale which provides the macroscopic information for the cellular automaton models, huge amounts of computational resources will be consumed by the numerical solution of the coupled system. Although a series of powerful parallel algorithms are developed to improving the computational efficiency, it is still difficult for the cellular automaton methods to simulate the microstructure evolution with a more fine mesh for accurate solution of details as a result of unreasonable computation time and inadequate computer memory.

\section{Conclusions}

The modeling and simulation of the solid-state diffusional transformations in metals are reviewed and the results may be summarized as follows:

- The traditional diffusion-controlled and interface-controlled modes fail to correctly describe the growth kinetics of new phase over the entire course of the transformation. The mixed-mode model which can consider both the effects and the varying relative importance of two extreme kinetics modes provides an appropriate approach to describe the character of actual phase transformation accurately.

- The nucleation, the growth of new phase with diffusionand mixed-mode character, and the impingement effects of the growing neighboring grains are described briefly.

- Aiming at the precision prediction of the nucleation, the growth and the impingement in the transformation, the developments of the empirical and analytical models, the phase-field models, the cellular automaton models, the Monte Carlo models and the molecular dynamics methods are summarized in detailed. Five numerical techniques provide good tools for the simulation of complex microstructure evolution.

- By comparing with these models, it is found that: the empirical and analytical models only provide average information on macroscale; the mechanistic-based phase field simulation can predict the evolution of arbitrary complex microstructure without any presumption on their shape or mutual distribution and explicitly tracking the of the interface position while it is more computationally intensive; the cellular automata models are more easily implemented and possess the high computational efficiency while its lack physical meanings on the time scale and the growth kinetics of grains; the Monte Carlo model as usually is very similar to the cellular automata model; the molecular dynamics simulations are utilized to describe atomic interactions to make up for the disadvantage of mesoscopic models while the representative volume element that can be simulated is very limited.

- Increasing efforts will be expected, to focus on exploring more advanced numerical simulation techniques coupled with atomistic calculations and macroscopic simulation, for predicting many important or non-negligible phenomena such as the mixture of different nucleation modes, the anisotropic growth caused by the anisotropic 
interfacial energy or mobility, the hard and soft impingement, and the effect of the thermal history and the elastic or plastic deformation.

The authors would like to gratefully acknowledge the project supported by the National Natural Science Foundation of China (51675433), and the Research Fund of the State Key Laboratory of Solidification Processing (NWPU), China (Grant No.149-QZ2016) and the 111 Project (B08040).

\section{References}

1. Y.J. Lan et al., Mesoscale simulation of deformed austenite decomposition into ferrite by coupling a cellular automaton method with a crystal plasticity finite element model, Acta Materialia 53 (2005) 991-1003

2. H. Yang et al., Review on cellular automata simulations of microstructure evolution during metal forming process: Grain coarsening, recrystallization and phase transformation, Sci. China Technol. Sci. 54 (2011) 2107-2118

3. Y.H. Jiang et al., Solid-state phase transformation kinetics in the near-equilibrium regime, J. Mater. Sci. 50 (2015) 662677

4. K.J. Song et al., Cellular automaton-based study of factors that affect dynamic solid phase transformation kinetics, Appl. Math. Model. 39 (2015) 5058-5072

5. H. Li et al., Non-isothermal phase-transformation kinetics model for evaluating the austenization of $55 \mathrm{CrMo}$ steel based on Johnson-Mehl-Avrami equation, Materials \& Design 92 (2016) 731-741

6. Y.H. Jiang, F. Liu, S.J. Song, Extension of analytical model of solid-state phase transformation, Trans. Nonferrous Metals Soc. China 22 (2012) 1176-1181

7. M. Meng et al., On the modeling of diffusion-controlled growth of primary alpha in heat treatment of two-phase Tialloys, J. Alloys \& Compounds 691 (2016) 67-80

8. R. Shi et al., Microstructure and transformation texture evolution during $\alpha$, precipitation in polycrystalline $\alpha / \beta$, titanium alloys - A simulation study, Acta Materialia 94 (2015) 224-243

9. S. Cui et al., Interface stress evolution of martensitic transformation in $\mathrm{MnCu}$ alloys: a phase-field study, Materials \& Design 109 (2016) 88-97

10. Y. Deng, S. Xiu, Research on microstructure evolution of austenitization in grinding hardening by cellular automata simulation and experiment, Int. J. Adv. Manuf. Technol. 93 (2017) 2599-2612

11. C. Halder, L. Madej, M. Pietrzyk, Discrete micro-scale cellular automata model for modelling phase transformation during heating of dual phase steels, Archives Civil \& Mech. Eng. 14 (2014) 96-103

12. D.S. Svyetlichnyy, Three-dimensional frontal cellular automata model of microstructure evolution phase transformation module, ISIJ Int. 54 (2014) 1386-1395

13. B.J. Kooi, Monte Carlo simulations of phase transformations caused by nucleation and subsequent anisotropic growth: extension of the Johnson-Mehl-Avrami-Kolmogorov theory, Phys. Rev. B 70 (2004) 155-163

14. H. Song, J.J. Hoyt, A molecular dynamics study of heterogeneous nucleation at grain boundaries during solidstate phase transformations, Comput. Mater. Sci. 117 (2016) 151-163
15. G.Z. Quan, J. Pan, Z.H. Zhang, Phase transformation and recrystallization kinetics in space-time domain during isothermal compressions for Ti-6Al-4V analyzed by multifield and multi-scale coupling FEM, Materials \& Design 94 (2016) 523-535

16. N.M. Xiao et al., Progress in mesoscopic modeling of microstructure evolution in steels, Sci. China Technol. Sci. 55 (2012) 341-356

17. N. Moelans, B. Blanpain, P. Wollants, An introduction to phase-field modeling of microstructure evolution, CalphadComput. Coupling Phase Diagrams \& Thermochemistry 32 (2008) 268-294

18. M.A. Miodownik, A review of microstructural computer models used to simulate grain growth and recrystallisation in aluminium alloys, J. Light Metals 2 (2002) 125-135

19. H. Hallberg, Approaches to modeling of recrystallization, Metals 1 (2011) 16-48

20. J.M. Haile, Molecular dynamics simulation: elementary methods, Comput. Phys. 7 (1993) 625

21. J. Svoboda et al., Kinetics of interfaces during diffusional transformations, Acta Materialia 49 (2001) 1249-1259

22. A.K. Jena, M.C. Chaturvedi, Phase transformation in materials, Prentice Hall, 1992

23. W.A. Soffa, D.E. Laughlin, Diffusional phase transformations in the solid state. Phys. Metall. (2014) 0851-1020

24. E. Kozeschnik, Modeling solid-state diffusion - Computational Materials Engineering - 5. Comput. Mater. Eng., 2007 151-177

25. H.I. Aaronson, M. Enomoto, J.K. Lee, Mechanisms of diffusional phase transformations in metals and alloys, 2010

26. J. Sietsma, S.V.D. Zwaag, A concise model for mixed-mode phase transformations in the solid state, Acta Materialia 52 (2004) 4143-4152

27. C. Zener, Theory of growth of spherical precipitates from solid solution, J. Appl. Phys. 20 (1949) 950-953

28. J.W. Christian, The theory of transformations in metals and alloys, Newnes, 2002

29. J. Sietsma et al., Evolution of the mixed-mode character of solid-state phase transformations in metals involving solute partitioning, Zeitschrift Für Metallkunde 97 (2006) $356-361$

30. E.D. Schmidt, E.B. Damm, S.A. Sridhar, Study of diffusionand interface-controlled migration of the austenite/ferrite front during austenitization of a case-hardenable alloy steel, Metall. \& Mater. Trans. A 38 (2007) 698-715

31. H. Chen, B. Appolaire, S.V.D. Zwaag, Application of cyclic partial phase transformations for identifying kinetic transitions during solid-state phase transformations: experiments and modeling, Acta Materialia 59 (2011) $6751-6760$

32. M. Hillert, Solute drag, solute trapping and diffusional dissipation of Gibbs energy 1, Acta Materialia 47 (1999) 4481-4505

33. G.P. Krielaart, J. Sietsma, S.V.D. Zwaag, Ferrite formation in $\mathrm{Fe}-\mathrm{C}$ alloys during austenite decomposition under nonequilibrium interface conditions, Mater. Sci. \& Eng. A 237 (1997) 216-223

34. A. Salwén, A new model for diffusional growth, Metallurgical Transactions A 24 (1993) 1507-1516

35. G. Eriksson, K. Hack, ChemSage - a computer program for the calculation of complex chemical equilibria, Metall. Trans. B 21 (1990) 1013-1023

36. J.W. Gibbs, On the equilibrium of heterogeneous substances, Trans Connecticut Acad, 1967 
37. R. Becker, W. Döring, Kinetische Behandlung der Keimbildung in übersättigten Dämpfen, Annalen Der Physik 416 (1935) 719-752

38. J.W. Cahn, J.E. Hilliard, Free energy of a nonuniform system. I. Interfacial free energy, J. Chem. Phys. 28 (1958) 258-267

39. J.W. Cahn, J.E. Hilliard, Free energy of a nonuniform system, III. Nucleation in a two-component incompressible fluid. The Selected Works of John W. Cahn, 2013, pp. 39-50

40. R. Poduri, L.Q. Chen, Non-classical nucleation theory of ordered intermetallic precipitates - application to the Al- $\mathrm{Li}$ alloy, Acta Materialia 44 (1996) 4253-4259

41. D.A. Porter, K.E. Easterling, Phase transformations in metal and alloys, 2nd edition. Chapman \& Hall, 1992

42. F. Liu, F. Sommer, E.J. Mittemeijer, An analytical model for isothermal and isochronal transformation kinetics, J. Mater. Sci. 39 (2004) 1621-1634

43. F. Liu et al., Analysis of solid state phase transformation kinetics: models and recipes, Metall. Rev. 52 (2013) 193-212

44. M. Avrami, Kinetics of phase change. II Transformationtime relations for random distribution of nuclei, J. Chem. Phy. 8 (1940) 212-224

45. M. Avrami, Granulation, phase change, and microstructure kinetics of phase change. III, J. Chem. Phys. 9 (1941) 177-184

46. Y. Liu et al., Isothermal austenite-ferrite transformation of Fe -0.04 at.\% C alloy: dilatometric measurement and kinetic analysis, Acta Materialia 56 (2008) 3833-3842

47. G. Meyrick, G.W. Powell, Phase transformations in metals and alloys, Annu. Rev. Mater. Sci. 74 (2003) 299-301

48. T.W. Heo, L.Q. Chen, Phase-field modeling of nucleation in solid-state phase transformations, JOM 66 (2014) 1520-1528

49. Y.V. Leeuwen, Moving interfaces in low-carbon steel - a phase transformation model, Appl. Sci. 29 (2000) 223-230

50. R.D. Doherty, Diffusive phase transformations in the solid state - Physical Metallurgy (Fourth Edition) - Chapter 15 [J]. Phys. Metall. (1996) 1363-1505

51. Ø. Grong, H.R. Shercliff, Microstructural modelling in metals processing, Progr. Mater. Sci. 47 (2002) 163-282

52. H.S. Carslaw, J.C. Jaeger, Conduction of heat in solids/by H. S. Carslaw and J. C. Jaeger[J], 1959

53. H.B. Aaron, D. Fainstein, G.R. Kotler, Diffusion-limited phase transformations: a comparison and critical evaluation of the mathematical approximations, J. Appl. Phys. 41 (1970) 4404-4410

54. S.L. Semiatin et al., Microstructure evolution during alphabeta heat treatment of Ti-6Al-4V, Metall. \& Mater. Trans. A 34 (2003) 2377-2386

55. M. Avrami, Kinetics of phase change. I general theory, J. Chem. Phys. 7 (1939) 1103-1112

56. K. Fan et al., Analysis of soft impingement in nonisothermal precipitation, J. Mater. Res. 24 (2009) 3664-3673

57. C. Wert, C. Zener, Interference of growing spherical precipitate particles, J. Appl. Phys. 21 (1950) 5-8

58. K. Fan et al., Modeling of isothermal solid-state precipitation using an analytical treatment of soft impingement,Acta Materialia 56 (2008) 4309-4318

59. S.E. Offerman et al., Solid-state phase transformations involving solute partitioning: modeling and measuring on the level of individual grains, Acta Materialia 52 (2004) 4757-4766

60. D. Crespo et al., Microstructural evaluation of primary crystallization with diffusion-controlled grain growth, Phys. Rev. B Condens. Matter 55 (1997) 3435-3444
61. C. Hao, S.V.D. Zwaag, Modeling of soft impingement effect during solid-state partitioning phase transformations in binary alloys, J. Mater. Sci. 46 (2011) 1328-1336

62. M. Tomellini, Soft impingement in diffusion-controlled growth of binary alloys: moving boundary effect in one-dimensional system, J. Mater. Sci. 48 (2013) $5653-5663$

63. H. Chen, V.D.Z. Sybrand, A mixed-mode model considering soft impingement effects for solid-state partitioning phase transformations, Solid State Phenom. 172-174 (2011) 561-566

64. R.E. Lyon, An integral method of nonisothermal kinetic analysis, Thermochimica Acta 297 (1997) 117-124

65. J. Málek, Crystallization kinetics by thermal analysis, J. Therm. Anal \& Calorim. 56 (1999) 763-769

66. W.A. Johnson, Reaction kinetics in process of nucleation and growth, Trans. AIME 135 (1939) 416-458

67. S.J. Song et al., Kinetics of solid-state transformation subjected to anisotropic effect: model and application, Acta Materialia 59 (2011) 3276-3286

68. F. Liu et al., Analytical description for solid-state phase transformation kinetics: extended works from a modular model, a review, J. Mater. Sci. \& Technol. 32 (2016) 97-120

69. V.V. Sessa, M. Fanfoni, M. Tomellini, Validity of Avrami's kinetics for random and nonrandom distributions of germs, Phys. Rev. B Condens. Matter 54 (1996) 836-841

70. G. Tegze et al., Diffusion-controlled anisotropic growth of stable and metastable crystal polymorphs in the phase-field crystal model, Phys. Rev. Lett. 103 (2009) 035702

71. J.W. Cahn, The kinetics of grain boundary nucleated reactions, Acta Metallurgica 4 (1956) 449-459

72. K.F. Kelton, A.L. Greer, C.V. Thompson, Transient nucleation in condensed systems, J. Chem. Phys. 79 (1983) 6261-6276

73. M. Tomellini, M. Fanfoni, M. Volpe, Spatially correlated nuclei: how the Johnson-Mehl-Avrami-Kolmogorov formula is modified in the case of simultaneous nucleation, Phys. Rev. B 62 (2000) 11300

74. P.R. Rios, E. Villa, Simultaneous and sequential transformations, Acta Materialia 59 (2011) 1632-1643

75. B.J. Kooi, Monte Carlo simulations of phase transformations caused by nucleation and subsequent anisotropic growth: extension of the Johnson-Mehl-Avrami-Kolmogorov theory, Physical Review B 70 (2004) 155-163

76. V.A. Shneidman, M.C. Weinberg, The effects of transient nucleation and size-dependent growth rate on phase transformation kinetics, J. Non-Crystalline Solids 160 (1993) 89-98

77. A. Bhattacharya, C.S. Upadhyay, S. Sangal, A quantitative phase-field simulation of soft-impingement in austenite to ferrite transformation with mixed-mode, Metall. \& Mater. Trans. A (2017) 1-14

78. M. Tomellini, Impingement factor in the case of phase transformations governed by spatially correlated nucleation, Phys. Rev. B 78 (2008) 1436-1446

79. M.C. Weinberg, D.P.B. Iii, Avrami exponents for transformations producing anisotropic particles, J. Non-Crystalline Solids 202 (1996) 290-296

80. B.J. Kooi. Extension of the Johnson-Mehl-AvramiKolmogorov theory incorporating anisotropic growth studied by Monte Carlo simulations, Phys. Rev. B 73 (2006) 054103 
81. A.T.W. Kempen, F. Sommer, E.J. Mittemeijer, The kinetics of the austenite-ferrite phase transformation of Fe-Mn: differential thermal analysis during cooling, Acta Materialia 50 (2002) 3545-3555

82. S.J. Song, F. Liu, Y.H. Jiang, Generalized additivity rule and isokinetics in diffusion-controlled growth, J. Mater. Sci. 49 (2014) 2624-2629

83. M. Hillert, Role of interfacial energy during solid-state phase transformations, Jernkontorets Annaler 141 (1957) 757-789

84. G.P. Ivantsov, The temperature field around a spherical, cylindrical, or pointed crystal growing in a cooling solution, Dokl. Akad. Nauk SSSR 58 (1947) 567-569

85. G. Horvay, J.W. Cahn, Dendritic and spheroidal growth, Acta Metallurgica 9 (1961) 695-705

86. R. Trivedi, The role of interfacial free energy and interface kinetics during the growth of precipitate plates and needles, Metall. Mater. Trans. B 1 (1970) 921-927

87. L.Q. Chen, W. Yang, Computer simulation of the domain dynamics of a quenched system with a large number of nonconserved order parameters: the grain-growth kinetics, Phys. Rev. B Condens. Matter 50 (1994) 15752

88. L.Q. Chen, A.G. Khachaturyan, Dynamics of simultaneous ordering and phase separation and effect of longrange Coulomb interactions, Phys. Rev. Lett. 70 (1993) 1477

89. J.D.V.D. Waals, The thermodynamic theory of capillarity under the hypothesis of a continuous variation of density, J. Stat. Phys. 20 (1979) 200-244

90. V.L. Ginzburg, L.D. Landau, On the theory of superconductivity, J. Exp. Theor. Phys. 20 (1950) 35

91. V.L. Ginzburg, L.D. Landau, Phenomenological theory, J. Exp. Theor. Phys. 20 (1950) 1064

92. L.Q. Chen, Phase-field models for microstructure evolution, Annu. Rev. Mater. Res. 32 (2002) 113-140

93. L.Q. Chen, Y. Wang, The continuum field approach to modeling microstructural evolution, JOM 48 (1996) 13-18

94. L. Zhang, L.Q. Chen, Q. Du, Simultaneous prediction of morphologies of a critical nucleus and an equilibrium precipitate in solids, Commun. Comput. Phys. 7 (2010) 674-682

95. T.W. Heo et al., Incorporating diffuse-interface nuclei in phase-field simulations, Scripta Materialia 63 (2010) $8-11$

96. W. Yan et al., Phase-field modeling of Widmanstätten ferrite formation during isothermal transformation in low carbon steels, Comput. Mater. Sci. 81 (2014) 503-509

97. T.W. Heo, L.Q. Chen, Phase-field modeling of nucleation in solid-state phase transformations, JOM 66 (2014) $1520-1528$

98. Y. Jin et al., 2D finite element modeling of misorientation dependent anisotropic grain growth in polycrystalline materials: level set versus multi-phase-field method, Comput. Mater. Sci. 104 (2015) 108-123

99. E. Miyoshi, T. Takaki, Extended higher-order multiphase-field model for three-dimensional anisotropicgrain-growth simulations, Comput. Mater. Sci. 120 (2016) 77-83

100. A.G. Khachaturyan, Theory of structural transformations in solids, Courier Corporation, 2013

101. F. Peter, P. Oliver, L.L. Joel, Modelling of phase separation in alloys with coherent elastic misfit, J. Stat. Phys. 95 (1999) 1429-1503
102. L. Q. Chen, S. Hu, Phase-field method applied to straindominated microstructure evolution during solid-state phase transformations. Continuum scale simulation of engineering materials: fundamentals-microstructures-process applications, 2004, pp. 271-296

103. B. Appolaire et al., Non-coherent interfaces in diffuse interface models, Philos. Magazine 90 (2010) 461-483

104. K. Ammar et al., Combining phase field approach and homogenization methods for modelling phase transformation in elastoplastic media, European J. Comput. Mech. 18 (2009) 485-523

105. K. Ammar et al., Modelling inheritance of plastic deformation during migration of phase boundaries using a phase field method, Meccanica 49 (2014) 2699-2717

106. V. De Rancourt et al., Homogenization of viscoplastic constitutive laws within a phase field approach, J. Mech. \& Phys. Solids 88 (2016) 291-319

107. J.P. Simmons et al., Microstructural development involving nucleation and growth phenomena simulated with the phase field method, Mater Sci. \& Eng. A 365 (2004) 136-143

108. G. Wang, D.C. Zeng, Z.W. Liu, Phase field study of concurrent nucleation and growth in a diffusion-controlled solid-state phase transformation, Adv. Mater. Res. 490495 (2012) 1140-1144

109. A. Yamanaka, T. Takaki, Y. Tomita, Multi-phase-field modeling of diffusive solid phase transition in carbon steel during continuous cooling transformation, J. Crys. Growth 310 (2008) 1337-1342

110. M. Militzer et al., Three-dimensional phase field modelling of the austenite-to-ferrite transformation, Acta Materialia 54 (2006) 3961-3972

111. Q. Chen et al., Quantitative phase field modeling of diffusion-controlled precipitate growth and dissolution in Ti-Al-V , Scripta Materialia 50 (2004) 471-476

112. N. Ofori-Opoku, N. Provatas, A quantitative multi-phase field model of polycrystalline alloy solidification, Acta Materialia 58 (2010) 2155-2164

113. I. Steinbach et al., CALPHAD and phase-field modeling: a successful liaison, J. Phase Equilibria \& Diffusion 28 (2007) 101-106

114. M. Militzer et al., Three-dimensional phase field modelling of the austenite-to-ferrite transformation, Acta Materialia 54 (2006) 3961-3972

115. S. Cui, J. Wan, X. Zuo et al., Three-dimensional, nonisothermal phase-field modeling of thermally and stressinduced martensitic transformations in shape memory alloys, Int. J. Solids \& Struc. 109 (2017) 1-11

116. I. Steinbach, F. Pezzolla, A generalized field method for multiphase transformations using interface fields, Physica D: Nonlinear Phenomena 134 (1999) 385-393

117. S.B. Biner, Programming phase- field modeling, Springer, 2017 T. Takaki et al., Two-dimensional phase- field simulations of dendrite competitive growth during the directional solidification of a binary alloy bicrystal, Acta Materialia 81 (2014) 272-283

118. T. Takaki et al., Two-dimensional phase-field simulations of dendrite competitive growth during the directional solidification of a binary alloy bicrystal, Acta Materialia 81 (2014) 272-283

119. C. Yang, Q. Xu, B. Liu, GPU-accelerated three-dimensional phase-field simulation of dendrite growth in a nickel-based superalloy, Comput. Mater. Sci. 136 (2017) 133-143 
120. Rolling of advanced high strength steels: theory, simulation and practice, CRC Press, 2017

121. K.G.F. Janssens. An introductory review of cellular automata modeling of moving grain boundaries in polycrystalline materials, Math. \& Comput. Simul. 80 (2010) 1361-1381

122. H.W. Hesselbarth, L. Kaps, F. Haessner, Two dimensional simulation of the recrystallization kinetics in the case of inhomogeneous stored energy. Mater. sci. Forum, (1993) 317-322

123. P.J. Hurley, F.J. Humphreys, Modelling the recrystallization of single-phase aluminium, Acta Materialia 51 (2003) 3779-3793

124. Y.C. Lin et al., Study of static recrystallization behavior in hot deformed Ni-based superalloy using cellular automaton model, Materials \& Design 99 (2016) 107-114

125. K.J. Song et al., Numerical simulation of $\beta$ to $\alpha$ phase transformation in heat affected zone during welding of TA15 alloy, Comput. Mater. Sci. 72 (2013) 93-100

126. C. Wu, Y. He, H.W. Li, Modeling of static coarsening of twophase titanium alloy in the $\alpha+\beta$ two-phase region at different temperature by a cellular automata method. Chin. Sci. Bull. 58 (2013) 3023-3032

127. M.F. Zhu, D.M. Stefanescu, Virtual front tracking model for the quantitative modeling of dendritic growth in solidification of alloys, Acta Materialia 55 (2007) 1741-1755

128. L. Madej, P.D. Hodgson, M. Pietrzyk, Multi-scale rheological model for discontinuous phenomena in materials under deformation conditions, Comput. Mater. Sci. 38 (2007) 685-691

129. J.L. He, M.T. Li, Cellular automata to simulate crack propagation of quasi-brittle materials, Appl. Mech. \& Mater. 90-93 (2011) 748-751

130. D.S. Svyetlichnyy, Modelling of the microstructure: from classical cellular automata approach to the frontal one, Comput. Mater. Sci. 50 (2011) 92-97

131. K.G.F. Janssens, Irregular cellular automata modeling of grain growth. Continuum scale simulation of engineering materials: fundamentals - microstructures - process applications, 2005, pp. 297-308

132. K.J. Song et al., Virtual front tracking cellular automaton modeling of isothermal $\beta$ to $\alpha$ phase transformation with crystallography preferred orientation of TA15 alloy, Model. \& Simul.Mater. Sci. \& Eng. 22 (2014) 5006

133. D. Raabe et al., Continuum scale simulation of engineering materials: fundamentals - microstructures - process applications, Front. Psychol. 6 (2014) 885

134. L. Beltran-Sanchez, D.M. Stefanescu, Growth of solutal dendrites: a cellular automaton model and its quantitative capabilities, Metall. \& Mater. Trans. A 34 (2003) 367-382

135. K.G.F. Janssens, E.A. Holm, S.M. Foiles, Introducing solute drag in irregular cellular automata modeling of grain growth, Mater. Sci. Forum 467-470 (2004) 1045-1050

136. D.S. Svyetlichnyy, Reorganization of cellular space during the modeling of the microstructure evolution by frontal cellular automata, Comput. Mater. Sci. 60 (2012) 153-162

137. D.S. Svyetlichnyy, Modeling of grain refinement by cellular automata, Comput. Mater. Sci. 77 (2013) 408-416

138. J.W. Cahn, The impurity-drag effect in grain boundary motion, Acta Metallurgica 10 (1962) 789-798
139. B. Su, Z. Han, B. Liu, Cellular automaton modeling of austenite nucleation and growth in hypoeutectoid steel during heating process, ISIJ Int. 53 (2013) 527-534

140. R. Golab et al., Sensivity analysis of the cellular automata model for austenite-ferrite phase transformation in steels, Appl. Math. 4 (2013) 1531-1536

141. C. Zheng, D. Raabe, D. Li, Prediction of post-dynamic austenite-to-ferrite transformation and reverse transformation in a low-carbon steel by cellular automaton modeling, Acta Materialia 60 (2012) 4768-4779

142. C. Zheng, D. Raabe, Interaction between recrystallization and phase transformation during intercritical annealing in a cold-rolled dual-phase steel: a cellular automaton model, Acta Materialia 61 (2013) 5504-5517

143. K.J. Song et al., Cellular automaton modeling of diffusion, mixed and interface controlled phase transformation, J. Phase Equilibria \& Diffusion 36 (2015) 136-148

144. F.Y. Wu, The Potts model, Rev. Modern Phys. 54 (1982) $235-268$

145. M.P. Anderson et al., Computer simulation of grain growth I. Kinetics, Acta Metallurgica 32 (1984) 783-791

146. A.D. Rollett, Overview of modeling and simulation of recrystallization, Prog. Mater. Sci. 42 (1997) 79-99

147. J.K. Mason, Grain boundary energy and curvature in Monte Carlo and cellular automata simulations of grain boundary motion, Acta Materialia 94 (2015) 162-171

148. B.J. Alder, T.E. Wainwright, Studies in molecular dynamics. I. General method, J. Chem. Phys. 31 (1959) 459-466

149. C.P. Chui et al., Molecular dynamics simulation of iron - a review, Spin 05 (2015)

150. S. Okita et al., Molecular dynamics simulations investigating consecutive nucleation, solidification and grain growth in a twelve-million-atom Fe-system, J. Cryst. Growth 474 (2017) 140-145

151. D.U. Furrer, Application of phase-field modeling to industrial materials and manufacturing processes, Current Opin. Solid State Mater. Sci. 15 (2011) 134-140

152. M. Mamivand, M.A. Zaeem, H.E. Kadiri, A review on phase field modeling of martensitic phase transformation, Comput. Mater. Sci. 77 (2013) 304-311

153. H. Li, X. Sun, H. Yang, A three-dimensional cellular automata-crystal plasticity finite element model for predicting the multiscale interaction among heterogeneous deformation, DRX microstructural evolution and mechanical responses in titanium alloys, Int. J. Plasti. 87 (2016) 154-180

154. M.A. Miodownik, A review of microstructural computer models used to simulate grain growth and recrystallisation in aluminium alloys, J. Light Metals 2 (2002) 125-135

155. P.W. Hoffrogge, L.A. Barrales-Mora, Grain-resolved kinetics and rotation during grain growth of nanocrystalline aluminium by molecular dynamics, Comput. Mater. Sci. 128 (2017) 207-222

156. S. Tateyama, Y. Shibuta, T. Suzuki, A molecular dynamics study of the fcc-bcc phase transformation kinetics of iron, Scripta Materialia 59 (2008) 971-974

157. S. Chakraborty, J. Zhang, S. Ghosh, Accelerated molecular dynamics simulations for characterizing plastic deformation in crystalline materials with cracks, Comput. Mater. Sci. 121 (2016) 23-34

Cite this article as: Xueyan Liu, Hongwei Li, Mei Zhan, A review on the modeling and simulations of solid-state diffusional phase transformations in metals and alloys, Manufacturing Rev. 5, 10 (2018) 